\title{
Radicamines A and B: Synthesis and Revision of the Absolute Configuration
}

\author{
Chu-Yi Yu*a and Mu-Hua Huang ${ }^{\mathrm{a}, \mathrm{b}}$ \\ Contribution from ${ }^{a}$ Beijing National Laboratory for Molecular Science (BNLMS), Laboratory for \\ Chemical Biology, Institute of Chemistry, Chinese Academy of Sciences, Beijing 100080, China \\ and ${ }^{b}$ Graduate University of the Chinese Academy of Sciences, Beijing 100049, China \\ yucy@iccas.ac.cn
}

Supporting Information

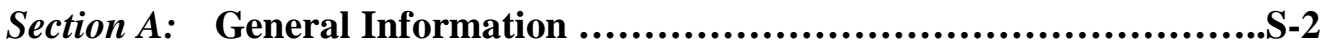

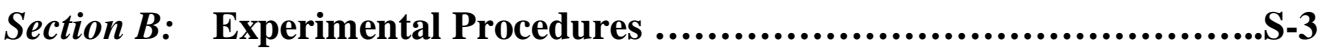

Section $C:{ }^{1} \mathrm{H},{ }^{13} \mathrm{C}$ and 2D-NMR Spectrum. ...............................S-13

Section D: $\quad$ References ...............................................S-42 


\section{Section A: General Information}

All reagents were used as received from commercial sources without further purification or prepared as described in the literature. Tetrahydrofuran was distilled from sodium and benzophenone immediately before use. Reactions were stirred using Teflon-coated magnetic stirring bars. Analytical TLC was performed with $0.20 \mathrm{~mm}$ silica gel $60 \mathrm{~F}$ plates with $254 \mathrm{~nm}$ fluorescent indicator. TLC plates were visualized by ultraviolet light or by treatment with a spray of Pancaldi reagent $\left\{\left(\mathrm{NH}_{4}\right)_{6} \mathrm{MoO}_{4}, \mathrm{Ce}\left(\mathrm{SO}_{4}\right)_{2}, \mathrm{H}_{2} \mathrm{SO}_{4}, \mathrm{H}_{2} \mathrm{O}\right\}$ or a solution $0.5 \%$ ninhydrin in acetone. Chromatographic purification of products was carried out by flash column chromatography on silica gel (230-400 mesh). Acidic ion exchange chromatography was performed on Amberlite ${ }^{\circledR}$ IR-120 $\left(\mathrm{H}^{+}\right)$or Dowex 50WX8-400, $\mathrm{H}^{+}$form. Melting points were determined using an electrothermal melting point apparatus. Both melting points and boiling points are uncorrected. Infrared spectra were recorded on a JASCO FT/IR-480 plus Fourier transform spectrometer. NMR spectra were measured in $\mathrm{CDCl}_{3}$ (with TMS as internal standard) or $\mathrm{D}_{2} \mathrm{O}$ on a Bruker $\mathrm{AV} 300\left({ }^{1} \mathrm{H}\right.$ at $300 \mathrm{MHz},{ }^{13} \mathrm{C}$ at $\left.75 \mathrm{MHz}\right)$ or a Bruker $\operatorname{AV} 600\left({ }^{1} \mathrm{H}\right.$ at $600 \mathrm{MHz},{ }^{13} \mathrm{C}$ at $\left.150 \mathrm{MHz}\right)$ magnetic resonance spectrometer. Chemical shifts $(\bar{\delta})$ are reported in ppm, and coupling constants $(J)$ are in Hz. The following abbreviations were used to explain the multiplicities: $\mathrm{s}=\operatorname{singlet}, \mathrm{d}=\operatorname{doublet}, \mathrm{t}=$ triplet, $\mathrm{q}=$ quartet, $\mathrm{m}=$ multiplet. High-resolution mass spectra (HRMS) were recorded on an APEXII FT-ICR (SIMS) mass spectrometer. Polarimetry was carried out using an Optical Activity AA-10R polarimeter and the measurements were made at the sodium D-line with a $0.5 \mathrm{dm}$ pathlength cell. Concentrations (c) are given in gram per $100 \mathrm{~mL}$.

\section{Section B: Experimental Procedures}

\section{$(3 S, 4 S, 5 R)$ - 5-Hydroxy-3,4,6-tribenzyloxy-1-hexene $13^{1}$}

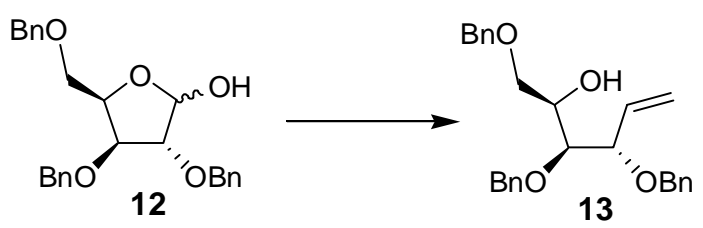

${ }^{\mathrm{n}}{ }^{-}$Butyllithium (1.6 M in hexane , $\left.34 \mathrm{ml}, 54.5 \mathrm{mmol}\right)$ was added dropwise to a stirred suspension of 
methyltriphenylphosphonium bromide $(19.6 \mathrm{~g}, 55 \mathrm{mmol})$ in dry $\mathrm{THF}(100 \mathrm{~mL})$ at about $0^{\circ} \mathrm{C}$ (ice-bath) in the atmosphere of Argon. The ice-bath was then removed and the resulted yellowish mixture was stirred for another 4 hours at room temperature. The reaction mixture was cooled with ice-water bath again, to which a solution of 2,3,5-O-tribenzyl-D-xylofuranose 12 (7.70 g, 18.3 mmol ) in THF $(60 \mathrm{~mL})$ was added dropwise. A cream-colored precipitate appeared, and the reaction mixture became difficult to stir. After stirring in room temperature for 12 hours, the mixture was heated for another 75 mins. The solvent was removed in vacuo, the residue was fully distributed between ethyl acetate and water. The combined ethyl acetate phase was dried and concentrated, and purified by flash column chromatography (silica gel, petroleum ether/AcOEt = 4:1) to give the title compound $\mathbf{1 3}$ as a light yellow oil (5.90 g, yield 77\%).

$[\alpha]_{\mathrm{D}}^{20}=-10.7\left(\mathrm{c}=0.56, \mathrm{CHCl}_{3}\right)$ for its enantiomer $[\alpha]_{\mathrm{D}}^{25}=+5.8\left(\mathrm{c}=1.6, \mathrm{CHCl}_{3}\right)$ in Pearson, W. H. ; Hines, J. V. J. Org. Chem 2000, 65, 5785-5793, \}. IR (cm $\left.{ }^{-1}\right)$ : 3447(w), 3064(w), 3030(w), 2864(w), 1507(m), 1496(s), 1091(vs), 1062(vs).ESI-MS: m/z 419.4 [M+H] ${ }^{+} ; 441.4[\mathrm{M}+\mathrm{Na}]^{+}$. HRMS (SIMS): m/z 419.2218 $[\mathrm{M}+\mathrm{H}]^{+},\left(\mathrm{C}_{27} \mathrm{H}_{31} \mathrm{O}_{4}\right.$ requires 419.2217) .

\section{(3S,4R,5R)-5-Methanesulfonyloxy-3,4,6-tribenzyloxy-1-hexene 14 and}

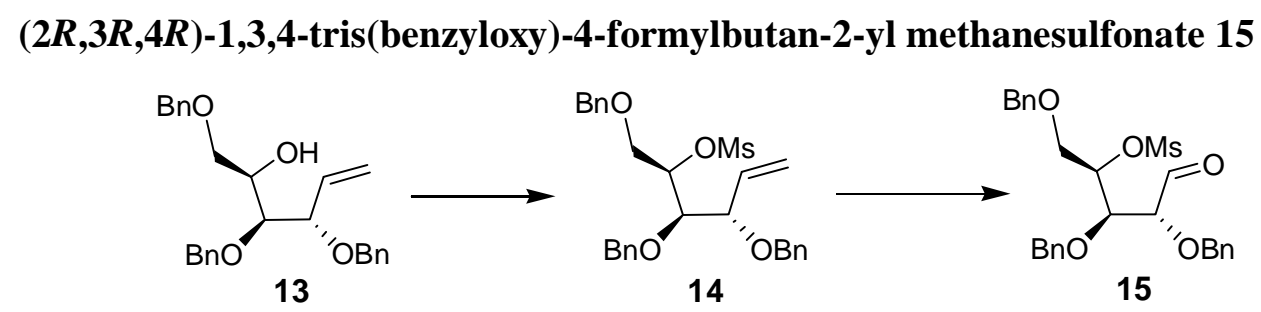

Pyridine (10ml) was added to a solution of (3S,4S,5R)- 5-hydroxy-3,4,6-tribenzyloxy-1-hexene 13 (5.90 g, $14 \mathrm{mmol})$ in DCM $(10 \mathrm{ml})$. The resulting solution was cooled with ice-bath and added dropwise with methanesulphonyl chloride $(2.0 \mathrm{ml}, 25.7 \mathrm{mmol})$. The reaction mixture was stirred at room temperature for about 10 hours. Then the solvent was evaporated in vacuo, the residue was purified by flash column chromatography ( mesylate 14 as a light yellow oil (6.56 g, yield 94\%).

$[\alpha]_{\mathrm{D}}^{20}=+15.6\left(\mathrm{c}=0.64, \mathrm{CHCl}_{3}\right)$. IR $(\mathrm{cm}-1): 3063(\mathrm{w}), 3031(\mathrm{w}), 2979(\mathrm{w}), 2936(\mathrm{w}), 1496(\mathrm{w})$, 1454(m), 1358(vs), 1174(vs), 1115(s), 1088(s), 1066(s), 1028(s). ${ }^{1} \mathrm{H}-\mathrm{NMR}\left(\mathrm{CDCl}_{3}, 300 \mathrm{M}\right): \delta$ (ppm): 7.34-7.24 (m, $15 \mathrm{H}, \mathrm{ArH}), 5.96-5.85(\mathrm{~m}, 1 \mathrm{H}, \mathrm{CH}=), 5.39(\mathrm{dd}, 2 \mathrm{H}, J=9.3$ and $9.6 \mathrm{~Hz})$, 
$4.88(\mathrm{dt}, 1 \mathrm{H}, J=2.9,3.0$, and $8.9 \mathrm{~Hz}), 4.68(\mathrm{ABQ}, 2 \mathrm{H}, J=11.3 \mathrm{~Hz}), 4.41(\mathrm{ABQ}, 2 \mathrm{H}, J=11.7$ $\mathrm{Hz}), 4.38(\mathrm{ABQ}, 2 \mathrm{H}, J=11.8 \mathrm{~Hz}), 3.97(\mathrm{dd}, 1 \mathrm{H}, J=4.3$ and $7.4 \mathrm{~Hz}), 3.67(\mathrm{dd}, 1 \mathrm{H}, J=2.9$ and $11.4 \mathrm{~Hz}), 3.54(\mathrm{dd}, 1 \mathrm{H}, J=5.8$ and $11.3 \mathrm{~Hz}), 2.90(\mathrm{~s}, 3 \mathrm{H}) .{ }^{13} \mathrm{C}-\mathrm{NMR}\left(\mathrm{CDCl}_{3}, 75 \mathrm{M}\right): \delta(\mathrm{ppm})$ $38.40\left(\mathrm{CH}_{3}\right), 69.19\left(\mathrm{CH}_{2} \mathrm{O}\right), 70.53\left(\mathrm{OCH}_{2}\right), 73.29\left(\mathrm{OCH}_{2}\right), 75.35\left(\mathrm{OCH}_{2}\right), 79.37(\mathrm{CHO}), 80.03$ (CHO), $81.86(\mathrm{CHO}), 119.98\left(=\mathrm{CH}_{2}\right), 127.82,127.91,127.97,128.28,128.38,128.44,128.50$, 129.04, 129.79, $134.49(\mathrm{CH}=), 137.49$ (C), $137.71(\mathrm{C}), 137.76(\mathrm{C}) . \mathrm{ESI}-\mathrm{MS}: \mathrm{m} / \mathrm{z} 519.4$ [M+Na] HRMS (SIMS) : m/z 497.1984 $[\mathrm{M}+\mathrm{H}]^{+}\left(\mathrm{C}_{28} \mathrm{H}_{33} \mathrm{O}_{6} \mathrm{~S}\right.$, requires 497.1992); 519.1805 [M+Na] $\left(\mathrm{C}_{28} \mathrm{H}_{33} \mathrm{O}_{6} \mathrm{SNa}\right.$, requires 519.1811 .

A solution of the alkene-mesylate $14(550 \mathrm{mg}, 1.1 \mathrm{mmol})$ in DCM $(50 \mathrm{~mL})$ was cooled with cold acetone bath $\left(-78^{\circ} \mathrm{C}\right)$ and bubbled with $\mathrm{O}_{3}$ in $\mathrm{O}_{2}$ atmophere for 7 mins. TLC indicated the disappearance of the starting material. The unreacted $\mathrm{O}_{3}$ was driven off by purging the system with $\mathrm{N}_{2}$. Dimethyl sulfide $(0.24 \mathrm{ml})$ was then added to the reaction mixture. After stirring for 3 hours, the solvent was removed in vacuo. The light yellow residue (800mg) was purified by flash chromatography (silica gel, petroleum ether/AcOEt $=2: 1$ ) to give the title compound $\mathbf{1 5}$ as an colorless oil (415mg, yield $76 \%)$.

$[\alpha]_{\mathrm{D}}^{20}=+30.3^{0}\left(\mathrm{c}=1.1, \mathrm{CHCl}_{3}\right) . \mathrm{IR}(\mathrm{cm}-1): 3031(\mathrm{w}), 2936(\mathrm{w}), 2871(\mathrm{w}), 1732(\mathrm{~s}), 1496(\mathrm{w}), 1454(\mathrm{~m})$, 1358(vs), 1175(vs), 1092(s). DEPT-135 $\left(\mathrm{CDCl}_{3}, 75 \mathrm{M}\right): \delta(\mathrm{ppm})$ positive : 201.82, 128.67, 128.58, $128.47,128.42,128.32,128.07,128.01,81.49,80.26,77.34,38.25$. negative : $74.75,73.45,68.44$. ${ }^{13} \mathrm{C}-\mathrm{NMR}\left(\mathrm{CDCl}_{3}, 75 \mathrm{M}\right): \delta(\mathrm{ppm}) 201.82,137.26,136.80,136.40,128.67,128.58,128.47,128.42$, 128.32, 128.07, 128.01, 81.49, 80.26, 77.34, 74.75, 73.45, 68.44, 38.25. ESI-MS: m/z 521.4 $[\mathrm{M}+\mathrm{Na}]^{+} . \mathrm{HRMS}(\mathrm{SIMS}): \mathrm{m} / \mathrm{z} 521.1609[\mathrm{M}+\mathrm{Na}]^{+}\left(\mathrm{C}_{27} \mathrm{H}_{30} \mathrm{O}_{7} \mathrm{SNa}\right.$, requires 521.1604$)$.

\section{$(3 S, 4 S, 5 S)-3,4-D i b e n z y l o x y-5-b e n z y l o x y m e t h y l-1-p y r r o l i n e-N-o x i d e ~ 9^{2}$}

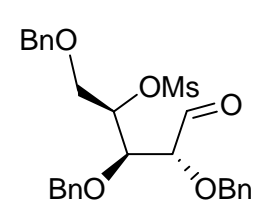

15

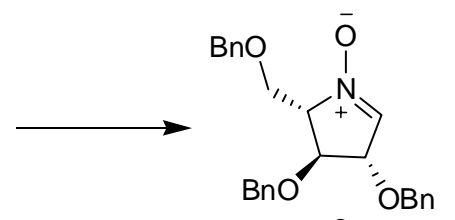

9

A solution of sodium carbonate $(5.09 \mathrm{~g}, 48 \mathrm{mmol})$ in $\mathrm{H}_{2} \mathrm{O}(7 \mathrm{ml})$ was added to a suspension of 
hydroxylamine hydrogen chloride $(3.36 \mathrm{~g}, 48 \mathrm{mmol})$ in $\mathrm{MeOH}(20 \mathrm{ml})$. The resulting mixture $(\mathrm{pH}$ $=8.0)$ was added with a solution of the mesylate-aldehyde 15 (8.03 g, $16 \mathrm{mmol})$ in THF (6 ml) and $\mathrm{MeOH}(8 \mathrm{ml})$. The reaction mixture was stirred and heated (at 50-60 ${ }^{\circ} \mathrm{C}$, oil bath) for 79 hours. Then water and AcOEt was added and the two phases were separated. The AcOEt phase was washed with water and the aqueous layer was extracted with AcOEt several times until TLC revealed no more products remaining in the aqueous solution. The combined organic phase was dried $\left(\mathrm{Na}_{2} \mathrm{SO}_{4}\right)$ and concentrated. The residue was purified by flash column chromatography (silicon gel, petroleum ether/AcOEt: $2: 1$ ) to give the target nitrone 9 as a white solid (4.03g, yield $60 \%)$.

m.p. $90-91^{\circ} \mathrm{C} \cdot[\alpha]_{\mathrm{D}}{ }^{20}=+45^{\circ}\left(\mathrm{c}=0.4, \mathrm{CHCl}_{3}\right)\left\{\right.$ Lit. $^{2 \mathrm{a}}$ ent-9: m.p. $80-82^{\circ} \mathrm{C} \cdot[\alpha]_{\mathrm{D}}{ }^{19}=-41.7^{\circ}(\mathrm{c}=1.00$, $\left.\mathrm{CHCl}_{3}\right)$; Lit. $^{2 \mathrm{~b}}$ ent-9: m.p. $\left.91-93^{\circ} \mathrm{C} .[\alpha]_{\mathrm{D}}{ }^{19}=-42^{\circ}\left(\mathrm{c}=1.3, \mathrm{CHCl}_{3}\right)\right\}$. IR $\left(\mathrm{cm}^{-1}\right): 3049(\mathrm{w}), 2945$, 2923, 2901, 2884, 2868, 2851(w), 1593 (s), 1551(s), 1496(s), 1452(s), 1361(s), 1247(vs),1131(vs), 1247(vs), 1028 (vs). ${ }^{1} \mathrm{H}-\mathrm{NMR}\left(\mathrm{CDCl}_{3}\right): \delta$ (ppm) 7.38- $7.26(\mathrm{~m}, 15 \mathrm{H}), 6.91$ (d, J=1.9 Hz, $\left.1 \mathrm{H}\right)$, 4.69-4.67 (m, 1 H), 4.64-4.46 (m, 6 H), 4.39 (dd, J=3.2, 2.2 Hz, 1 H), 4.10-4.04 (m, 2 H), 3.78 (d, $J=7.3 \mathrm{~Hz}, 1 \mathrm{H}) .{ }^{13} \mathrm{C}-\mathrm{NMR}\left(\mathrm{CDCl}_{3}, 75 \mathrm{M}\right): \delta(\mathrm{ppm}) 66.03\left(\mathrm{CH}_{2} \mathrm{O}\right), 71.67,71.91,73.47,80.30(\mathrm{CHO})$, 82.74 (CHO), 127.70, 127.75, 127.92, 128.14, 128.17, 128.38, 128.55, 128.61, $133.02(\mathrm{~N}=\mathrm{CH})$, $137.06(\mathrm{C}), 137.16(\mathrm{C}), 137.63(\mathrm{C}) .\left[\mathrm{lit}:{ }^{13} \mathrm{C}\right.$ NMR: $\delta$ (ppm) 137.7 (s), 137.2 (s), 137.1 (s), 132.9 (d), 128.6, 128.5, 128.4, 128.1, 127.9 and 127.7 (d, 15 C), 82.7 (d), $80.3(\mathrm{~d}), 77.4(\mathrm{~d}), 73.9(\mathrm{t}), 71.9(\mathrm{t})$, 71.6 (t), 66.1 (t). ]. ESI-MS : m/z 440.3 [M+Na] $]^{+}$. HRMS (SIMS): m/z 418.2007 [M+H] $\left(\mathrm{C}_{26} \mathrm{H}_{28} \mathrm{NO}_{4}\right.$ requires 418.2013$)$.

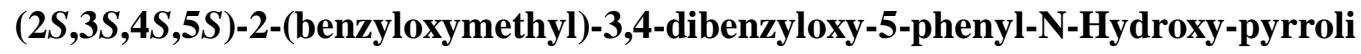

\section{dine 17}

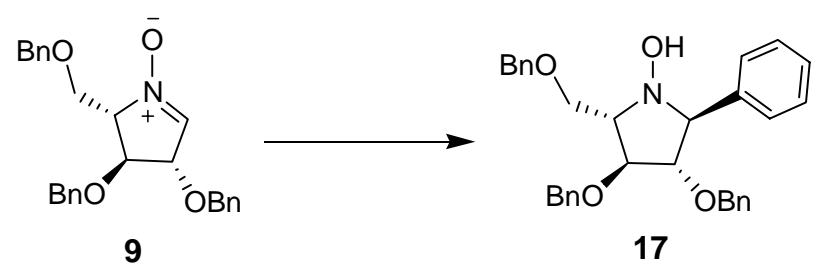

To a solution of phenylmagnesium bromide in THF [prepared by heating Mg turnings (60 mg, 2.5 $\mathrm{mmol})$ and bromobenzene $(0.26 \mathrm{ml}, 2.5 \mathrm{mmol})$ in hot dry $\mathrm{THF}]$ was added dropwise with a solution of cyclic nitrone $9\left(200 \mathrm{mg}\llcorner 0.5 \mathrm{mmol})\right.$ in THF $(6 \mathrm{ml})$ at about $0^{\circ} \mathrm{C}$ (ice-water bath). The reaction mixture was stirred overnight (about 12 h.), quenched with sat. aqueous $\mathrm{NaHCO}_{3}$ solution and extracted with diethyl ether and AcOEt. The combined extracts were dried and solvents were 
removed in vacuo. The residue was purified by flash column chromatography (silica gel, petroleum ether/AcOEt: 4/1) to give the title compound $\mathbf{1 7}$ as an colorless oil, which was dried under vacuum to give a white solid (216mg, yield $91 \%)$.

m.p. $81-82^{\circ} \mathrm{C} .[\alpha]_{D}=+16.8\left(\mathrm{c}=2.5, \mathrm{CHCl}_{3}\right) . \operatorname{IR}(\mathrm{cm}-1): 3343(\mathrm{w}), 3107(\mathrm{w}), 3087(\mathrm{w}), 3063(\mathrm{w})$, 3031(w), 2971(w), 2870(w), 1604(w), 1595(w), 1496(w), 1472(w), 1454(m), 1365(w), 1098(s), 1076(vs). ${ }^{1} \mathrm{H}-\mathrm{NMR}\left(\mathrm{CDCl}_{3}\right): \delta(\mathrm{ppm}): 3.69-3.75(\mathrm{~m}, 1 \mathrm{H}), 3.78(\mathrm{t}, 1 \mathrm{H}, J=7.2 \mathrm{~Hz}), 3.89(\mathrm{dd}, 1$ $\mathrm{H}, J=3.7$ and $8.7 \mathrm{~Hz}), 4.06(\mathrm{dd}, 1 \mathrm{H}, J=3.4$ and $7.2 \mathrm{~Hz}), 4.11(\mathrm{dd}, 1 \mathrm{H}, J=2.7$ and $3.2 \mathrm{~Hz})$, $4.23(\mathrm{~d}, 1 \mathrm{H}, J=7.2 \mathrm{~Hz}), 4.33(\mathrm{ABQ}, 2 \mathrm{H}, J=11.8 \mathrm{~Hz}), 4.55(\mathrm{ABQ}, 2 \mathrm{H}, J=12.0 \mathrm{~Hz}), 4.56(\mathrm{ABQ}$ $2 \mathrm{H}, J=12.1 \mathrm{~Hz}), 4.94(\mathrm{~s}, 1 \mathrm{H}, \mathrm{OH}), 7.07-7.44(\mathrm{~m}, 20 \mathrm{H}) \cdot{ }^{1} \mathrm{H}-\mathrm{NMR}\left(\mathrm{CDCl}_{3}+\mathrm{D}_{2} \mathrm{O}\right): \delta(\mathrm{ppm}):$ 3.73-3.77 (m, $1 \mathrm{H}), 3.80(\mathrm{t}, 1 \mathrm{H}, J=7.2 \mathrm{~Hz}), 3.93(\mathrm{dd}, 1 \mathrm{H}, J=3.9$ and $8.9 \mathrm{~Hz}), 4.09(\mathrm{dd}, 1 \mathrm{H}, J$ $=3.4$ and $7.2 \mathrm{~Hz}), 4.14(\mathrm{dd}, 1 \mathrm{H}, J=2.8$ and $3.2 \mathrm{~Hz}), 4.26(\mathrm{~d}, 1 \mathrm{H}, J=7.2 \mathrm{~Hz}), 4.37(\mathrm{ABQ}, 2 \mathrm{H}$, $J=11.9 \mathrm{~Hz}), 4.60(\mathrm{~s}, 2 \mathrm{H}), 4.58(\mathrm{ABQ}, 2 \mathrm{H}, J=12.0 \mathrm{~Hz}), 7.12-7.47(\mathrm{~m}, 20 \mathrm{H})$. DEPT-135 $\left(\mathrm{CDCl}_{3}, 75 \mathrm{M}\right): \delta(\mathrm{ppm})$ positive $: 128.50,128.38,128.34,128.23,128.05,127.78,127.69,127.67$ 127.62, 127.56, 87.49, 83.68, 74.06, 68.93. negative : 73.44, 72.01, 71.66, 66.86.

${ }^{13} \mathrm{C}-\mathrm{NMR}\left(\mathrm{CDCl}_{3}, 75 \mathrm{M}\right): \delta(\mathrm{ppm}) 139.36,138.25,138.11,137.89,128.49,128.38,128.34,128.23$, $128.05,127.77,127.69,127.67,127.61,127.55,87.50,83.69,74.07,73.44,72.01,71.66$, 68.93,66.87. ESI-MS : m/z 496.5 [M+H] ${ }^{+}$. HRMS (SIMS) : m/z 496.2485 [M+H] ${ }^{+},\left(\mathrm{C}_{32} \mathrm{H}_{33} \mathrm{NO}_{4}\right.$ requires 496.2482$)$.

\section{(2S,3S,4S,5S)-2-(hydroxymethyl)-5-phenylpyrrolidine-3,4-diol 18}

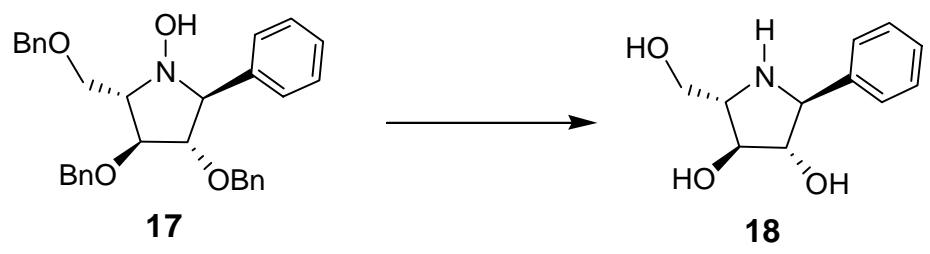

$10 \mathrm{ml}$ of $\mathrm{MeOH}$ and $1 \mathrm{ml}$ of $\mathrm{HCl}(6 \mathrm{~N})$ was added to the solution of the hydroxylamine 17 (200 mg, $0.4 \mathrm{mmol})$ in DCM (10mL). To which $\mathrm{Pd} / \mathrm{C}(10 \%, 60 \mathrm{mg})$ was added under stirring. After hydrogenation under atmospheric pressure for 20 hours, TLC (AcOEt/MeOH: 5/1) revealed the formation of a polar compound. The system was bubbled with $\mathrm{N}_{2}$, and the $\mathrm{Pd} / \mathrm{C}$ was filtered off. 
After the solution was concentrated in vacuo, the residue was dissolved in $\mathrm{MeOH}$ and neutralized with con. aqueous ammonium solution. The resulting solution was concentrated in vacuo. The residue was dried under vacuum and purified by an acidic ion exchanger column (Dowex 5Wx8-400, $\mathrm{H}^{+}$form, Aldrich, column size: $\left.1.3 \times 14 \mathrm{~cm}\right)$, eluting with distilled water $(50 \mathrm{ml})$ and then $1 \mathrm{~N} \mathrm{NH}{ }_{4} \mathrm{OH}$, affording the title compound 18 as a white solid (72 mg, yield 85\%).

$[\alpha]_{\mathrm{D}}=-52^{0}\left(\mathrm{c}=0.65, \mathrm{H}_{2} \mathrm{O}\right) .{ }^{1} \mathrm{H}-\mathrm{NMR}\left(\mathrm{D}_{2} \mathrm{O}, 300 \mathrm{M}\right): \delta(\mathrm{ppm}): 7.41(\mathrm{~m}, 5 \mathrm{H}), 4.09(\mathrm{dd}, 1 \mathrm{H}, J=7.4$ and 8.3 Hz), 3.93 (broad t, 2H), $3.72(\mathrm{dd}, 1 \mathrm{H}, J=4.7$ and $11.6 \mathrm{~Hz}), 3.65(\mathrm{dd}, 1 \mathrm{H}, \mathrm{J}=6.3$ and $11.6 \mathrm{~Hz}$ ), $3.22(\mathrm{dd}, 1 \mathrm{H}, J=6.3$ and $11.5 \mathrm{~Hz}) .{ }^{1} \mathrm{H}-\mathrm{NMR}\left(\mathrm{D}_{2} \mathrm{O}, 600 \mathrm{M}\right): \delta(\mathrm{ppm}): 7.36($ broad d, 4H, H-2', H-3', H-5', H-6'), 7.32-7.27 (m, 1H, H-4'), 4.04 (dd, 1H, J=7.5 and 9.0 Hz, H-4), 3.88 (d, 1H, J=5.6 Hz, H-3 or H-5), 3.87 (d, 1H, J=7.4 Hz, H-5 or H-3), 3.67 (dd, 1H, J=4.5 and $11.6 \mathrm{~Hz}, \mathrm{H}-1 \mathrm{a}), 3.61$ (dd, $1 \mathrm{H}, J=6.5$ and $11.6 \mathrm{~Hz}, \mathrm{H}-1 \mathrm{~b}), 3.17(\mathrm{dd}, 1 \mathrm{H}, J=2.1$ and $6.8 \mathrm{~Hz}, \mathrm{H}-2)$. DEPT-135 $\left(\mathrm{D}_{2} \mathrm{O}, 75 \mathrm{M}\right): \delta$ (ppm) positive : $128.95,128.23,127.35,82.26,77.51,64.00,61.79$. negative : $62.46 .{ }^{13} \mathrm{C}-\mathrm{NMR}$ $\left(\mathrm{D}_{2} \mathrm{O}, 75 \mathrm{M}\right): \delta(\mathrm{ppm}) 139.73,128.96,128.23,127.36,82.27,77.53,64.01,62.47,61.81$. ESI-MS : $\mathrm{m} / \mathrm{z} 210.2[\mathrm{M}+\mathrm{H}]^{+}, 232.2[\mathrm{M}+\mathrm{Na}]^{+}$. HRMS (SIMS) : m/z $210.1126[\mathrm{M}+\mathrm{H}]^{+},\left(\mathrm{C}_{11} \mathrm{H}_{16} \mathrm{NO}_{3}\right.$ requires 210.1125$)$.

\section{2-(benzyloxy)-4-bromo-1-methoxybenzene $23^{3}$}

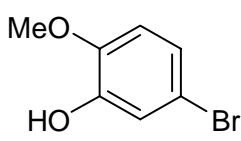

22

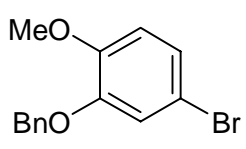

23

To a suspension of sodium hydride $(0.95 \mathrm{~g}, 55 \%$ in mineral oil, $21.7 \mathrm{mmol})$ in THF (10 ml) was added a solution of 5-bromo-2-methoxyphenol $22(4.0 \mathrm{~g}, 19.7 \mathrm{mmol})$ in THF (10 ml). TBAT (0.2 g, $0.5 \mathrm{mmol}$ ) was then added to the reaction mixture, followed by addition of a solution of benzyl bromide $(2.6 \mathrm{ml}, 21.7 \mathrm{mmol})$ in THF $(5 \mathrm{ml})$. The reaction mixture was stirred at $50-60^{\circ} \mathrm{C}$. When TLC indicated the disappearance of the starting material, the reaction mixture was cooled down to room temperature and was poured into ice-water. The white solids precipitating from the solution were collected, filtered and recrystallized from AcOEt/petroleum ether (1:1) to give $\mathbf{2 3}$ as colorless needles (3.40 g, yield 90\%) . 
m.p. $106-107^{\circ} \mathrm{C}$ (in lit $\left.70-71^{\circ} \mathrm{C}\right) .{ }^{1} \mathrm{H}$ NMR $\left(\mathrm{CDCl}_{3}, 300 \mathrm{MHz}\right): \delta$ (ppm) $3.88(3 \mathrm{H}, \mathrm{s}), 5.14$ (2H. s), $6.79(1 \mathrm{H}, J=8.8 \mathrm{~Hz}), 7.06-7.09(2 \mathrm{H}, \mathrm{m}), 7.32-7.48(5 \mathrm{H}, \mathrm{m}) .{ }^{13} \mathrm{C} \mathrm{NMR}\left(\mathrm{CDCl}_{3}, 75 \mathrm{MHz}\right): \delta(\mathrm{ppm})$ $56.2,71.2,112.6,113.1,117.3,124.0,127.4,128.1,128.6,136.4,149.0,149.1$;

(2S,3S,4S,5S)-2-(benzyloxymethyl)-3,4-dibenzyloxy-5-(3-benzyloxy-4-methoxyph enyl)-N-hydroxy-pyrrolidine 24

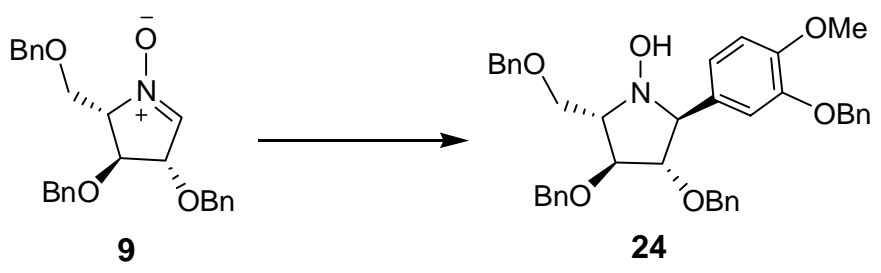

A solution of 2-(benzyloxy)-4-bromo-1-methoxybenzene 23 (0.73 g, $2.5 \mathrm{mmol})$ in THF (4 ml) was added to a mixture of magnesium turnings $(0.10 \mathrm{~g}, 4.2 \mathrm{mmol})$ in hot THF $(4 \mathrm{ml})$. The mixture was heated to reflux in the atmosphere of $\mathrm{N}_{2}$ until the magnesium disappeared and the grey solution formed. The Grignard reagent was cooled with an ice-water bath, and a solution of the cyclic nitrone 9 (300 mg, $0.72 \mathrm{mmol})$ in dry THF ( $5 \mathrm{ml})$ was added dropwise. The reaction mixture was stirred at room temperature overnight (about 12 h.), then quenched with sat. aqueous $\mathrm{NaHCO}_{3}$ solution, extracted with diethyl ether and AcOEt. The combined organic phase was dried (anhydrous $\mathrm{Na}_{2} \mathrm{SO}_{4}$ ) and solvents were removed in vacuo. The residue was purified by flash column chromatography (silica gel, petroleum ether/ACOEt: 4/1) to give the title compound 24 as an colorless oil, which turned into a white solid (400mg, yield $89 \%$ ) after being placed in a refrigerator.

$[\alpha]_{\mathrm{D}}=-4.4\left(\mathrm{c}=0.46, \mathrm{CHCl}_{3}\right) .{ }^{1} \mathrm{H}-\mathrm{NMR}\left(\mathrm{CDCl}_{3}, 300 \mathrm{M}\right): \delta(\mathrm{ppm}): 3.70-3.75(\mathrm{~m}, 1 \mathrm{H}), 3.79(\mathrm{dd}$, $1 \mathrm{H}, J=7.2$ and $9.1 \mathrm{~Hz}), 3.91(\mathrm{dd}, 1 \mathrm{H}, J=4.2$ and $8.9 \mathrm{~Hz}), 3.91(\mathrm{~s}, 3 \mathrm{H}), 3.98(\mathrm{dd}, 1 \mathrm{H}, J=3.5$ and 7.3 $\mathrm{Hz}), 4.12(\mathrm{dd}, 1 \mathrm{H}, J=3.2$ and $3.0 \mathrm{~Hz}), 4.16(\mathrm{~d}, 1 \mathrm{H}, J=7.3 \mathrm{~Hz}), 4.31(\mathrm{ABQ}, 2 \mathrm{H}, J=11.9 \mathrm{~Hz}), 4.58$ (ABQ, 2H, J=11.9 Hz), $4.60(\mathrm{~s}, 2 \mathrm{H}), 4.72(\mathrm{~s}$ and b ,1H, OH), $5.11(\mathrm{~s}, 3 \mathrm{H}), 6.88(\mathrm{~d}, 1 \mathrm{H}, J=8.2 \mathrm{~Hz})$ $6.99(\mathrm{dd}, 1 \mathrm{H}, J=1.8$ and $8.2 \mathrm{~Hz}), 7.04(\mathrm{~d}, 1 \mathrm{H}, J=1.8 \mathrm{~Hz}), 7.09-7.13(\mathrm{~m}, 2 \mathrm{H}), 7.25-7.45$ (m, 14H). ${ }^{13} \mathrm{C}-\mathrm{NMR}\left(\mathrm{CDCl}_{3}, 75 \mathrm{M}\right): \delta(\mathrm{ppm}) 56.1\left(\mathrm{OCH}_{3}\right), 67.0,68.7,70.9,71.7,72.0,73.5,73.7,83.7,87.4$, $111.6,113.7,121.5,127.5,127.6,127.7,127.8,128.0,128.2,128.4,128.4,128.5,131.8,137.1$, 137.9, 138.1, 138.2, 148.1, 148.4. Dept-135 $\left(\mathrm{CDCl}_{3}\right): \delta(\mathrm{ppm})$ positive :56.1, 68.7, 73.6, 83.7, 87.4, 
111.6, 113.7, 121.5, 127.5, 127.6, 127.7, 127.8, 128.0, 128.2, 128.4, 128.4, 128.5. negative: 67.0, 70.9, 71.7, 72.0, 73.5. HRMS (SIMS): m/z 632.3018 $[\mathrm{M}+\mathrm{H}]^{+},\left(\mathrm{C}_{40} \mathrm{H}_{32} \mathrm{NO}_{6}\right.$ requires 632.3006).

\section{$(2 S, 3 S, 4 S, 5 S)-2-(3-H y d r o x y-4-m e t h o x y-p h e n y l)-5-h y d r o x y m e t h y l-p y r r o l i d i n e-3,4$}

\section{-diol 1}

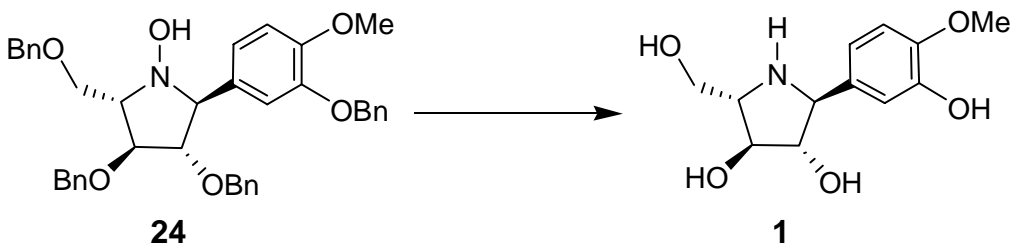

$\mathrm{MeOH}(10 \mathrm{ml})$ and $\mathrm{HCl}(6 \mathrm{~N}, 1 \mathrm{ml})$ were added to a solution of $24(80 \mathrm{mg}, 0.1 \mathrm{mmol})$ in DCM (10 m). To which $\mathrm{Pd} / \mathrm{C}(10 \%, 60 \mathrm{mg})$ was added under stirring. After hydrogenation under atmospheric pressure for 20 hours, TLC (AcOEt/MeOH: 5/1) revealed the formation of a polar compound. The system was bubbled with $\mathrm{N}_{2}$, and the $\mathrm{Pd} / \mathrm{C}$ was filtered off. After the solution was concentrated in vacuo, the residue $(52 \mathrm{mg})$ was dissolved in $\mathrm{MeOH}$ and neutralized with con. aqueous ammonium solution. The resulting solution was concentrated in vacuo. The residue was dried under vacuum and purified by an acidic ion exchanger column (Dowex $5 \mathrm{Wx} 8-400, \mathrm{H}^{+}$form, Aldrich, column size: $1.3 \times 14 \mathrm{~cm})$, eluting with distilled water $(50 \mathrm{ml})$ and then $1 \mathrm{~N} \mathrm{NH}_{4} \mathrm{OH}$, affording the title compound $\mathbf{1}$ as a yellow syrup (28 mg, yield $87 \%)$.

$[\alpha]_{D}=-44.4\left(\mathrm{c}=0.45, \mathrm{H}_{2} \mathrm{O}\right)\left\{\right.$ in the lit $\left.[\alpha]_{D}=+43.7\left(\mathrm{c}=0.13, \mathrm{H}_{2} \mathrm{O}\right)\right\} . \mathrm{IR}\left(\mathrm{cm}^{-1}\right): 3209(\mathrm{vs}), 1637(\mathrm{~m})$, 1401(vs), 1276(w), 1252(w), 1132(w), 1037(w). ${ }^{1} \mathrm{H}-\mathrm{NMR}\left(\mathrm{D}_{2} \mathrm{O}, 300 \mathrm{M}\right): \delta(\mathrm{ppm}): 6.99(\mathrm{~d}, 1 \mathrm{H}$, $J=8.1 \mathrm{~Hz}), 6.91(\mathrm{~s}, 1 \mathrm{H}), 6.88(\mathrm{dd}, 1 \mathrm{H}, J=5.1 \mathrm{~Hz}), 4.04(\mathrm{dd}, 1 \mathrm{H}, J=7.5,8.9 \mathrm{~Hz}), 3.91(\mathrm{t}, 1 \mathrm{H}, J=7.3 \mathrm{~Hz})$, $3.81\left(\mathrm{~s}, 3 \mathrm{H}, \mathrm{OCH}_{3}\right), 3.80(\mathrm{~d}, 1 \mathrm{H}, J=9.4 \mathrm{~Hz}), 3.72(\mathrm{dd}, 1 \mathrm{H}, J=4.7,11.6 \mathrm{~Hz}), 3.65$ (dd, 1H, $J=6.4$, $11.6 \mathrm{~Hz}), 3.21(\mathrm{dd}, 1 \mathrm{H}, J=6.4,11.1 \mathrm{~Hz}) .{ }^{1} \mathrm{H}-\mathrm{NMR}\left(\mathrm{D}_{2} \mathrm{O}, 600 \mathrm{M}\right): \delta(\mathrm{ppm}): 6.91(\mathrm{~d}, 1 \mathrm{H}, J=8.2 \mathrm{~Hz}$, H-5'), 6.84 (s, 1H, H-2'), 6.83 (d, 1H, J=8.5 Hz), 3.97 (t, 1H, J=7.8, 8.8Hz), 3.84 (t, 1H, J=7.3 Hz), 3.76-3.73 (d or t, 1H, H-5, overlap with $\left.\mathrm{OCH}_{3}\right), 3.74$ (s, 3H, $\left.\mathrm{OCH}_{3}\right), 3.64$ (dd, 1H, J=4.4, $11.6 \mathrm{~Hz}$, H-1a), 3.58 (dd, 1H, J=6.4 , 11.5 Hz, H-1b), 3.14 (dd, 1H, J=6.5, 11.2 Hz, H-2). DEPT 135 (D ${ }_{2} \mathrm{O}$, $75 \mathrm{MHz}$ ): $\delta(\mathrm{ppm})$ : positive : 119.65, 114.44, 112.75, 81.92, 77.34, 63.51, 61.68, 56.08. negative : 62.38. ${ }^{13} \mathrm{C}-\mathrm{NMR}\left(\mathrm{D}_{2} \mathrm{O}, 75 \mathrm{MHz}\right): \delta(\mathrm{ppm}) 147.42,145.43,132.63,119.66,114.45,112.77,81.93$, 
77.36, 63.52, 62.39, 61.69, 56.10. HRMS (SIMS) : m/z 256.1185 [M+H] $]^{+},\left(\mathrm{C}_{12} \mathrm{H}_{18} \mathrm{NO}_{5}\right.$ requires 256.1179).

The comparison of ${ }^{13} \mathrm{C}-\mathrm{NMR}$ of compound $\mathbf{1}$ (original lab note: $\mathbf{m h h} \mathbf{- 3 5 7 - p}$ ) with that of radicamine A isolated by Kusano (in Chem. Pharm. Bull. 2001,49,1362)

\begin{tabular}{|c|c|c|}
\hline $\begin{array}{c}\text { Radicamine } \mathrm{A}\left(\text { in } \mathrm{D}_{2} \mathrm{O} \text { ) }\right. \\
\text { isolated by Kusano }\end{array}$ & $\begin{array}{c}1 \text { (Mhh-357-p) } \\
\left(\text { in } \mathrm{D}_{2} \mathrm{O}\right)\end{array}$ & $\llcorner\delta$ \\
\hline $64.69(\mathrm{C}-1)$ & $62.39(\mathrm{C}-1)$ & 2.30 \\
\hline $64.15(\mathrm{C}-2)$ & $61.69(\mathrm{C}-2)$ & 2.46 \\
\hline $79.62(\mathrm{C}-3)$ & $77.36(\mathrm{C}-3)$ & 2.26 \\
\hline $84.16(\mathrm{C}-4)$ & $81.93(\mathrm{C}-4)$ & 2.23 \\
\hline $65.97(\mathrm{C}-5)$ & $63.52(C-5)$ & 2.45 \\
\hline 134.90 (C-1') & $132.63\left(\mathrm{C}-1^{\prime}\right)$ & 2.27 \\
\hline 116.95 (C-2’) & 114.45 (C-2’) & 2.50 \\
\hline 147.81 (C-3’) & 145.43 (C-3') & 2.38 \\
\hline 149.97 (C-4’) & 147.42 (C-4') & 2.55 \\
\hline $115.23\left(\mathrm{C}-5^{\prime}\right)$ & $112.77\left(\mathrm{C}-5^{\prime}\right)$ & 2.46 \\
\hline 122.35 (C-6’) & 119.66 (C-6’) & 2.69 \\
\hline $58.55\left(\mathrm{OCH}_{3}\right)$ & $56.10\left(\mathrm{OCH}_{3}\right)$ & 2.45 \\
\hline
\end{tabular}

\section{(2S,3S,4S,5S)-2-(Benzyloxymethyl)-3,4-dibenzyloxy-5-(4-benzyloxyphenyl)- $N$-hy} droxypyrrolidine 26 


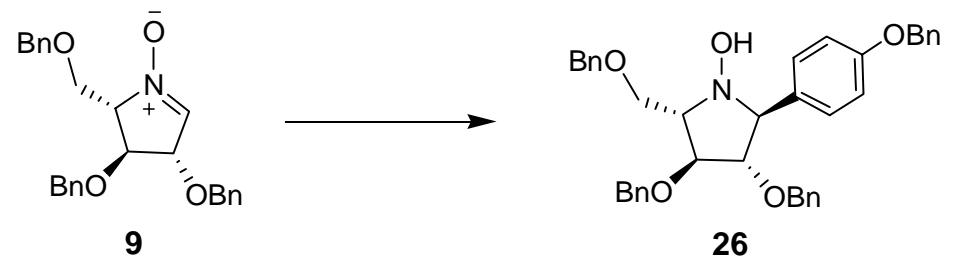

A mixture of $50 \mathrm{mg}$ ( $2 \mathrm{mmol})$ of $\mathrm{Mg}$ turnings and $500 \mathrm{mg}$ (2mmol) of 4-benzyloxy-bromobenzene ${ }^{4}$ in THF (4 ml) was heated and refluxed for 30 mins. The solution changed from yellow to brown, and was then cooled with an ice-water bath. The cold Grignard reagent was added dropwise to a solution of the cyclic nitrone $9(200 \mathrm{mg}, 0.5 \mathrm{mmol})$ in $\mathrm{THF}(15 \mathrm{ml})$ at about $0^{\circ} \mathrm{C}$ (ice-water bath). The reaction mixture was stirred overnight (about 12h.), then quenched with sat. aqueous $\mathrm{NaHCO}_{3}$ solution and extracted with diethyl ether. The combined extracts were dried (anhydrous $\mathrm{Na}_{2} \mathrm{SO}_{4}$ ) and solvents were removed in vacuo. The residue was purified by flash column chromatography (silica gel, petroleum ether/ACOEt: 4/1) to give the title compound $\mathbf{2 6}$ as a white solid (225 $\mathrm{mg}$, yield $80 \%)$.

m.p. : $129^{\circ} \mathrm{C} .[\alpha]_{\mathrm{D}}^{19}=+18.0\left(\mathrm{c}=0.55, \mathrm{CHCl}_{3}\right)$. IR(cm-1): 3136(s), 1613(w), 1518(w), 1454(w), 1401(vs), 1247(w), 1121(w), 1024(w). ${ }^{1} \mathrm{H}-\mathrm{NMR}\left(\mathrm{CDCl}_{3}, 300 \mathrm{M}\right): \delta(\mathrm{ppm}): 3.66-3.71(\mathrm{~m}, 1 \mathrm{H}$, $\mathrm{H}-2), 3.75(\mathrm{dd}, 1 \mathrm{H}, J=7.0$ and $9.0 \mathrm{~Hz}, \mathrm{H}-4), 3.84(\mathrm{dd}, 1 \mathrm{H}, J=3.9$, and $9.0 \mathrm{~Hz}, \mathrm{H}-3)$, 4.06-4.11 (m, $2 \mathrm{H}, \mathrm{H}-1), 4.20$ (d , 1H, $J=7.0 \mathrm{~Hz}, \mathrm{H}-5), 4.34$ (ABQ, 2H, $J=11.8$ and 19.0 Hz) , 4.56 (ABQ, 2 $\mathrm{H}, J=12.0 \mathrm{~Hz}), 4.55(\mathrm{ABQ}, 2 \mathrm{H}, J=12.0 \mathrm{~Hz}), 5.05(\mathrm{~s}, 2 \mathrm{H}), 6.94(\mathrm{~d}, 2 \mathrm{H}, J=8.7 \mathrm{~Hz}), 7.09(\mathrm{dd}$, $2 \mathrm{H}, J=3.6,2.3$ and $7.3 \mathrm{~Hz}), 7.21-7.45(\mathrm{~m}, 20 \mathrm{H}) .{ }^{13} \mathrm{C}-\mathrm{NMR}\left(\mathrm{CDCl}_{3}, 75 \mathrm{MHz}\right): \delta(\mathrm{ppm}) 66.8$, 68.9, 70.0 , 71.7, $72.0\left(\mathrm{CH}_{2} \mathrm{O}\right), 73.4,73.4(\mathrm{NCH}), 83.4,87.1(\mathrm{CHO}), 114.7,127.5,127.6,127.7$, $128.0,128.0,128.2,128.3,128.4,128.6,129.8(\mathrm{CH}), 137.0,137.8,138.0,138.1,158.5$. ESI-MS : m/z $624.3[\mathrm{M}+\mathrm{Na}]^{+}, 602.3[\mathrm{M}+\mathrm{H}]^{+}$. HRMS (SIMS): m/z $602.2899[\mathrm{M}+\mathrm{H}]^{+}$ $\left(\mathrm{C}_{39} \mathrm{H}_{40} \mathrm{NO}_{5}\right.$ requires 602.2901).

(2S,3S,4S,5S)-2-(hydroxymethyl)-5-(4-hydroxyphenyl)pyrrolidine-3,4-diol 2 

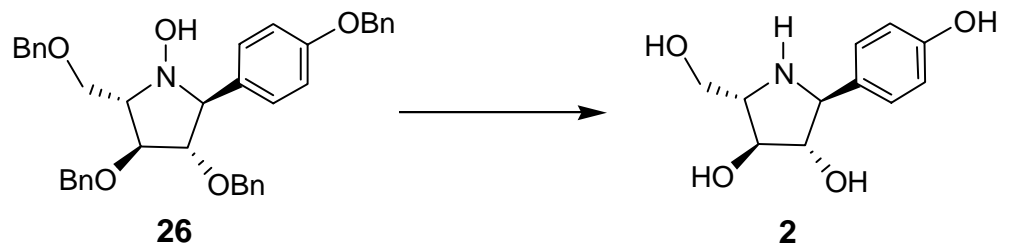

$10 \mathrm{ml}$ of acidic $\mathrm{MeOH}(200 \mathrm{~mL} / 2 \mathrm{~mL}: \mathrm{MeOH} / \mathrm{AcCl})$ was added to a solution of 26 (117 mg, 0.2 mmol) in DCM (5 ml). To which Pd/C (10\%, $60 \mathrm{mg})$ was added under stirring. After hydrogenation under atmospheric pressure for 20 hours, TLC (AcOEt/MeOH: 5/1) revealed the formation of a polar compound. The system was bubbled with $\mathrm{N}_{2}$, and the $\mathrm{Pd} / \mathrm{C}$ was filtered off. After the solution was concentrated in vacuo, the residue $(80 \mathrm{mg}$ ) was dissolved in $\mathrm{MeOH}$ and neutralized with con. aqueous ammonium solution. The resulting solution was concentrated in vacuo. The residue was dried under vacuum and purified by an acidic ion exchanger column (Dowex $5 \mathrm{Wx} 8-400, \mathrm{H}^{+}$form,

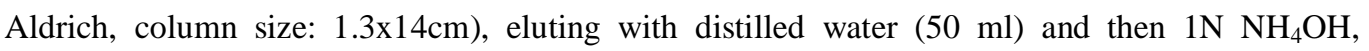
affording the title compound $\mathbf{2}$ as a yellow syrup (41 mg, yield $93 \%$ ).

$[\alpha]_{D}^{30}=-72.7\left(\mathrm{c}=0.17, \mathrm{H}_{2} \mathrm{O}\right)\left\{\right.$ in the lit $\left.[\alpha]_{\mathrm{D}}=+72.0\left(\mathrm{c}=0.10, \mathrm{H}_{2} \mathrm{O}\right)\right\} . \mathrm{IR}(\mathrm{cm}-1): 3199(\mathrm{vs}), 1617(\mathrm{~s})$, 1512(m), 1400(vs), 1260(w), 1179(w), 1125(w), 1074(w). ${ }^{1} \mathrm{H}-\mathrm{NMR}\left(\mathrm{D}_{2} \mathrm{O}, 300 \mathrm{M}\right): \delta(\mathrm{ppm}): 7.29$ $(\mathrm{d}, 1 \mathrm{H}, J=8.6 \mathrm{~Hz}), 6.87(\mathrm{~d}, 1 \mathrm{H}, J=8.6 \mathrm{~Hz}), 4.10(\mathrm{dd}, 1 \mathrm{H}, J=7.5,9.2 \mathrm{~Hz}, \mathrm{H}-4), 3.93(\mathrm{t}, 1 \mathrm{H}, J=7.4 \mathrm{~Hz}$ H-3), 3.90 (d, 1H, J=9.2 Hz, H-5), 3.74 (dd, 1H, J=4.6, 11.7 Hz, H-1a), 3.67 (dd, 1H, J=6.3, 11.7 $\mathrm{Hz}, \mathrm{H}-1 \mathrm{~b}), 3.28-3.22$ (m, 1H, H-2). ${ }^{1} \mathrm{H}-\mathrm{NMR}\left(\mathrm{D}_{2} \mathrm{O}, 600 \mathrm{M}\right): \delta(\mathrm{ppm}): 7.24$ (d, 1H, J=8.4 Hz, H-2', H-6’), 6.87 (d, 1H, J=8.4 Hz, H-3', H-5'), 4.08 (dd, 1H, J=7.8 and 9.2 Hz, H-4), 3.91 (d, 1H, J=7.2 Hz, H-5), 3.90 (dd, 1H, J=3.7 and 7.4Hz, H-3), 3.70 (dd, 1H, J=4.3, 11.8 Hz, H-1a), 3.64 (dd, 1H, $J=6.4,11.8 \mathrm{~Hz}, \mathrm{H}-1 \mathrm{~b}), 3.24(\mathrm{dd}, 1 \mathrm{H}, J=6.8$ and $11.2 \mathrm{~Hz}, \mathrm{H}-2)$. DEPT135 (D $2 \mathrm{O}, 300 \mathrm{MHz}): \delta$ $(\mathrm{ppm}):$ positive : $129.08,115.79,81.40,76.98,63.35,61.59$. negative $: 62.02 .{ }^{13} \mathrm{C}-\mathrm{NMR}\left(\mathrm{D}_{2} \mathrm{O}\right.$, $300 \mathrm{MHz}): \delta(\mathrm{ppm}) 155.77,130.48,129.08,115.79,81.40,76.98,63.35,62.02,61.59$. ESI-MS : m/z 226.2 $[\mathrm{M}+\mathrm{H}]^{+}$. HRMS (SIMS): m/z 226.1074 $[\mathrm{M}+\mathrm{H}]^{+}\left(\mathrm{C}_{11} \mathrm{H}_{16} \mathrm{NO}_{4}\right.$ requires 226.1074).

The comparison of ${ }^{13} \mathrm{C}-\mathrm{NMR}$ of compound $\mathbf{2}$ (original lab note: mhh-321-p) with that of radicamine B isolated by Kusano ${ }^{5}$ (in Chem. Pharm. Bull. 2001, 49, 1362) 


\begin{tabular}{|c|c|c|}
\hline $\begin{array}{c}\text { Radicamine B isolated by } \\
\text { Kusano }\left(\text { in } \mathrm{D}_{2} \mathrm{O}\right)\end{array}$ & $\begin{array}{c}\mathbf{2}(\mathbf{M h h}-321-\mathbf{p}) \\
\left(\text { in } \mathrm{D}_{2} \mathrm{O}\right)\end{array}$ & 2.33 \\
\hline $64.38(\mathrm{C}-1)$ & $62.05(\mathrm{C}-1)$ & 2.48 \\
\hline $64.12(\mathrm{C}-2)$ & $61.64(\mathrm{C}-2)$ & 2.32 \\
\hline $79.35(\mathrm{C}-3)$ & $77.03(\mathrm{C}-3)$ & 2.27 \\
\hline $83.72(\mathrm{C}-4)$ & $81.45(\mathrm{C}-4)$ & 2.47 \\
\hline $65.86(\mathrm{C}-5)$ & $63.39(\mathrm{C}-5)$ & 1.52 \\
\hline $131.78\left(\mathrm{C}-1^{\prime}\right)$ & $130.26\left(\mathrm{C}-1^{\prime}\right)$ & 2.58 \\
\hline $131.68\left(\mathrm{C}-2^{\prime}\right)$ & $129.10\left(\mathrm{C}-2^{\prime}\right)$ & 2.52 \\
\hline $118.34\left(\mathrm{C}-3^{\prime}\right)$ & $115.82\left(\mathrm{C}-3^{\prime}\right)$ & 2.51 \\
\hline $158.30\left(\mathrm{C}^{\prime} 4^{\prime}\right)$ & $155.79\left(\mathrm{C}-4^{\prime}\right)$ & 2.52 \\
\hline $118.34\left(\mathrm{C}-5^{\prime}\right)$ & $115.82\left(\mathrm{C}-5^{\prime}\right)$ & 2.58 \\
\hline $131.68\left(\mathrm{C}-6^{\prime}\right)$ & $129.10\left(\mathrm{C}-6^{\prime}\right)$ & \\
\hline
\end{tabular}

Section C: $\quad{ }^{1} \mathrm{H},{ }^{13} \mathrm{C}$ and 2D-NMR Spectrum 

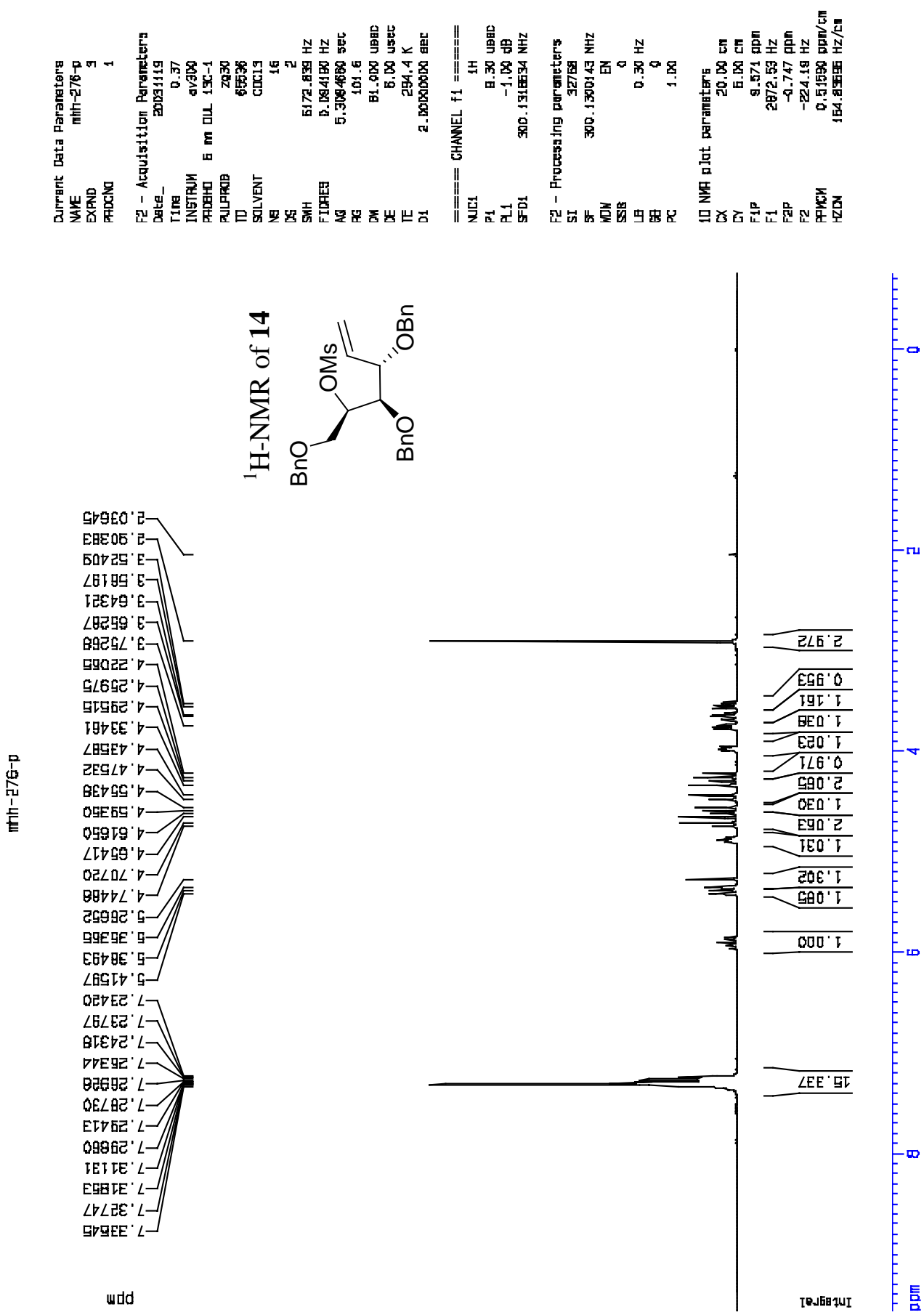

ป 


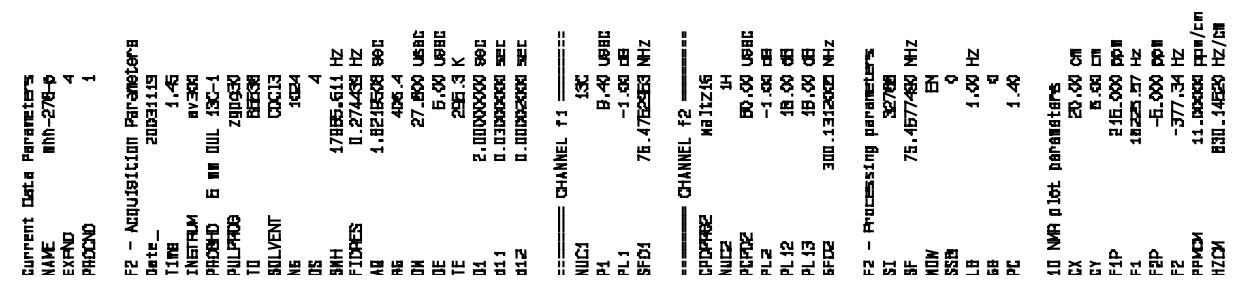

GGE 96<smiles>C=C1C(=O)OC(CO)C1O[Hg]</smiles>

981.69

$\angle 2 S^{\circ} \cdot \mathrm{OL}$

OSE $S L$

$6 \angle 9^{\circ} 9 \angle$

EOT $\angle L$

$\angle S^{\circ} \angle L-$

$\checkmark L E \cdot 6 L$

$\left.2 E 0^{\circ} \mathrm{OB}-1\right]$

BL6.6I -

OZg $\angle Z T-7$

БLE - $\angle 2 L]$

$10 \mathrm{\theta}$ ' $\theta \mathrm{CL}-$

ᄃ旺

$\angle E \nabla^{\circ}$ g己

$\angle$ ८०

9日7 $\nabla \mathrm{EL}$

Q66 " $\angle E T$

CTL' $\angle E V-$

GE $L^{\circ} \angle E I-$

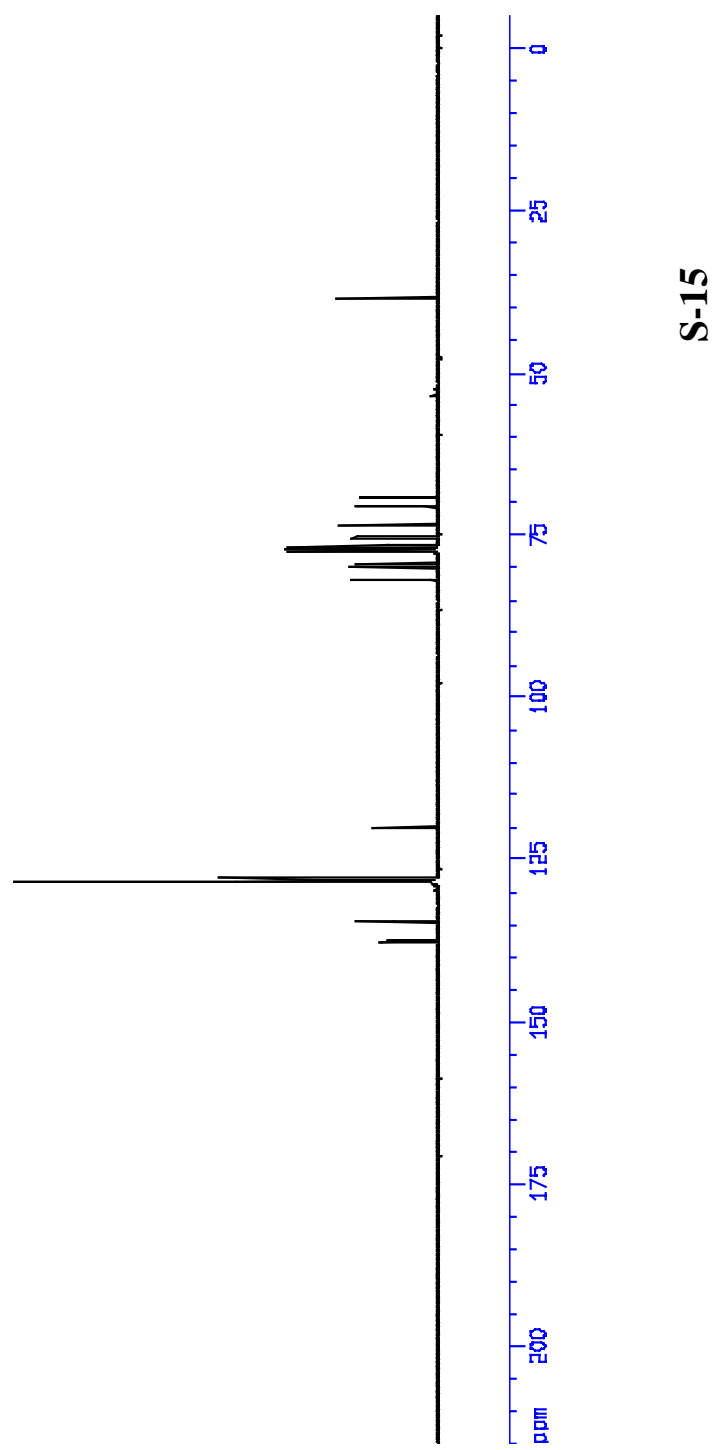



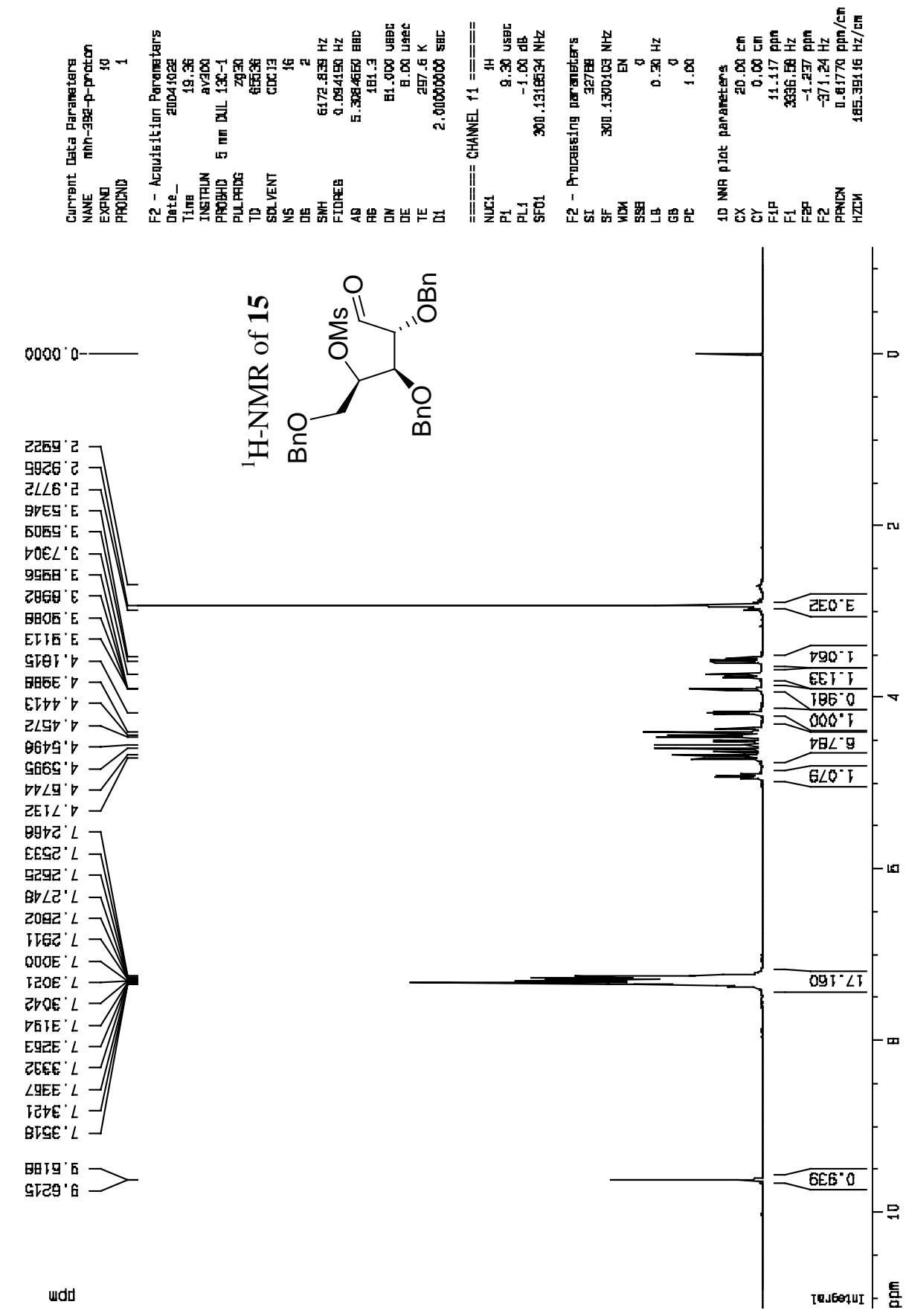

$\frac{1}{n}$ 


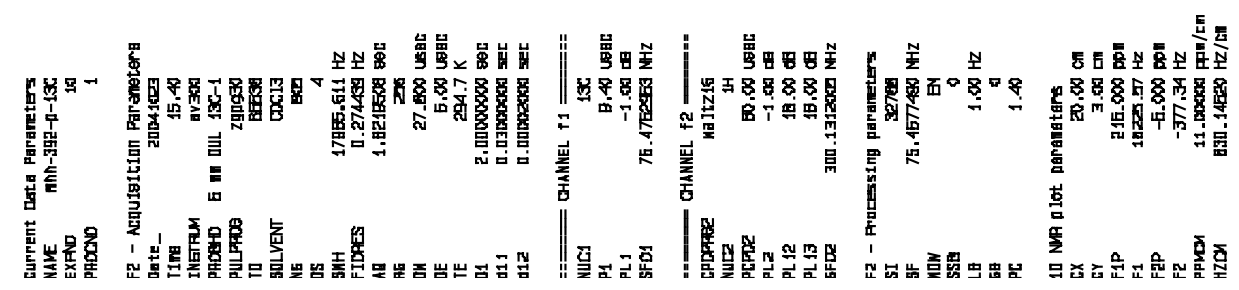

$9 v \mathrm{~V}^{-} \mathrm{EE}$
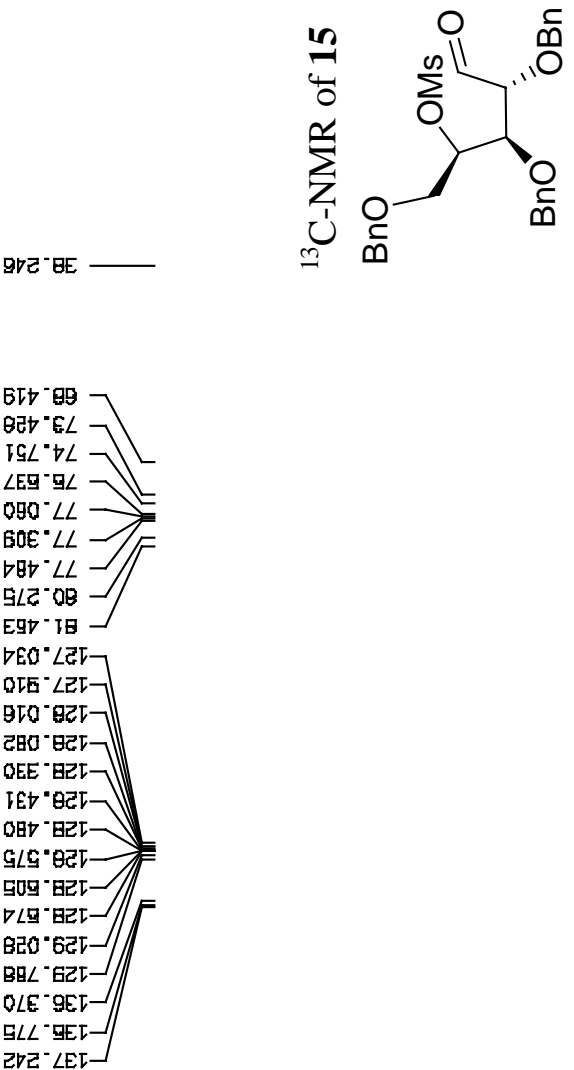

E99.102-

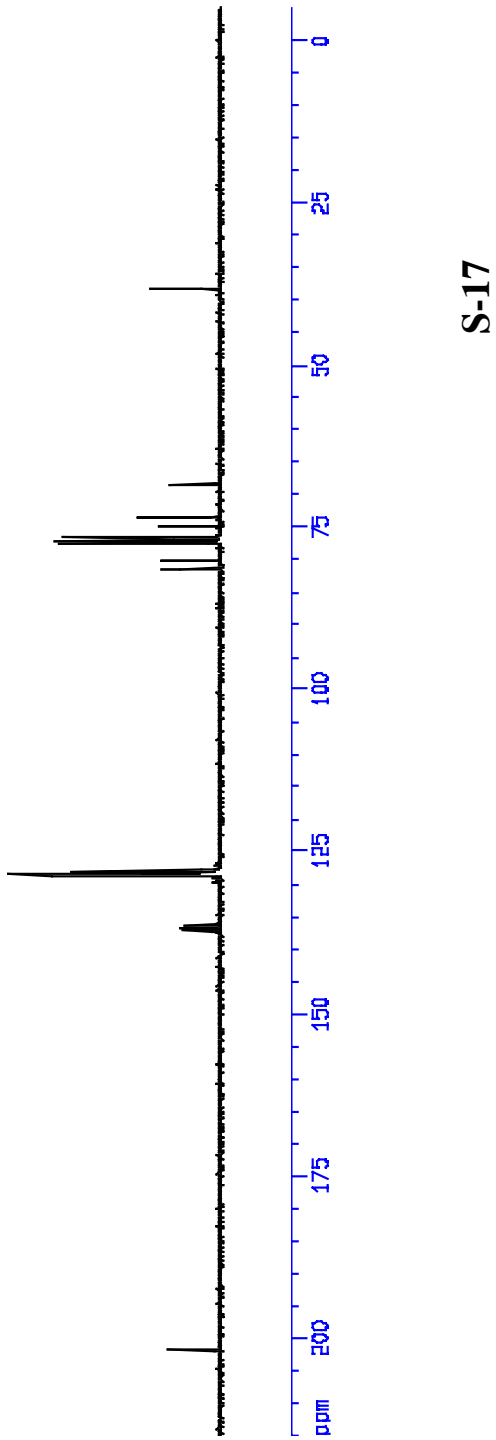




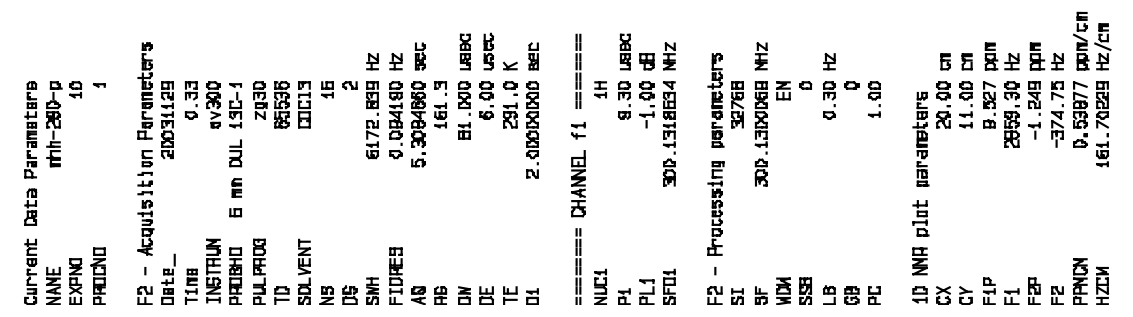

$50000^{\circ} 0$
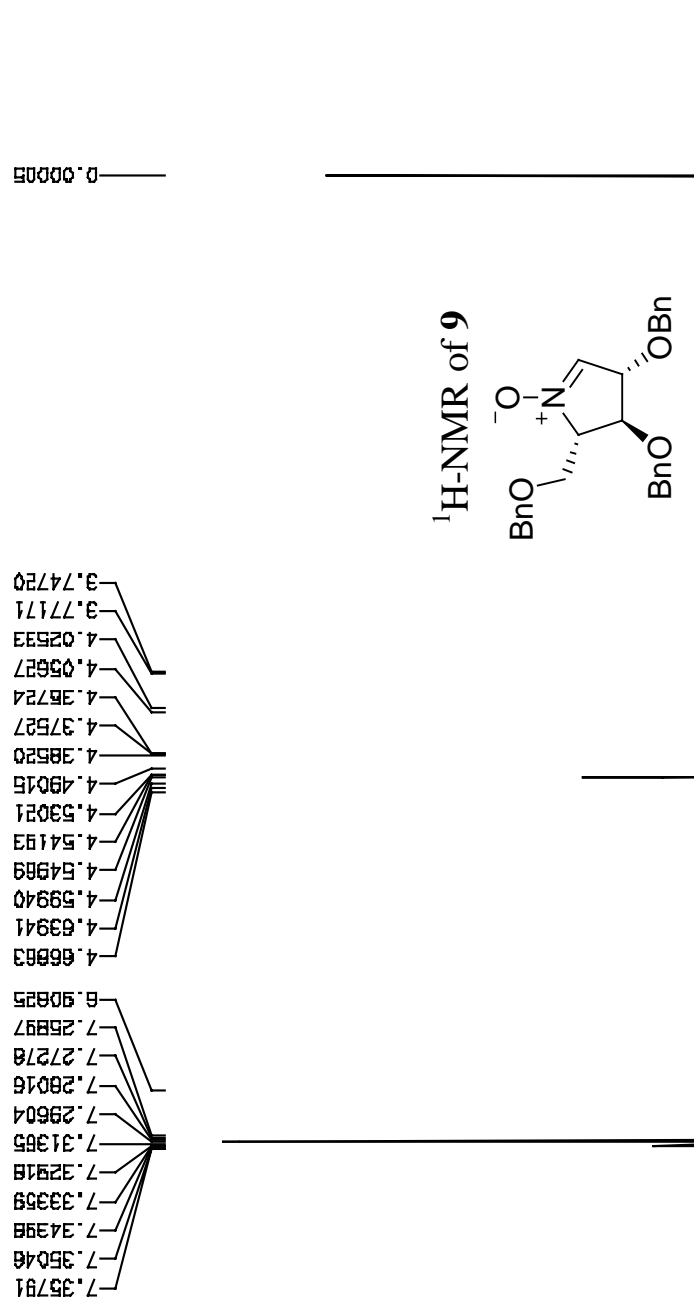

wda

$\frac{\infty}{n}$

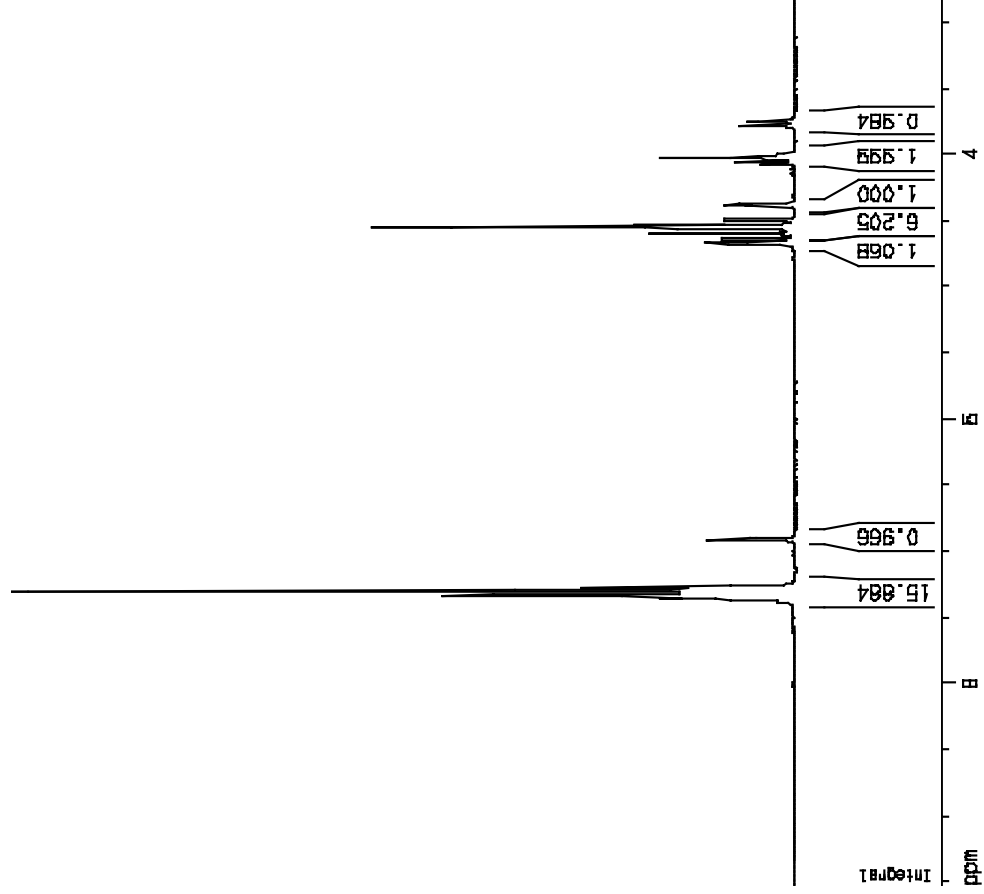




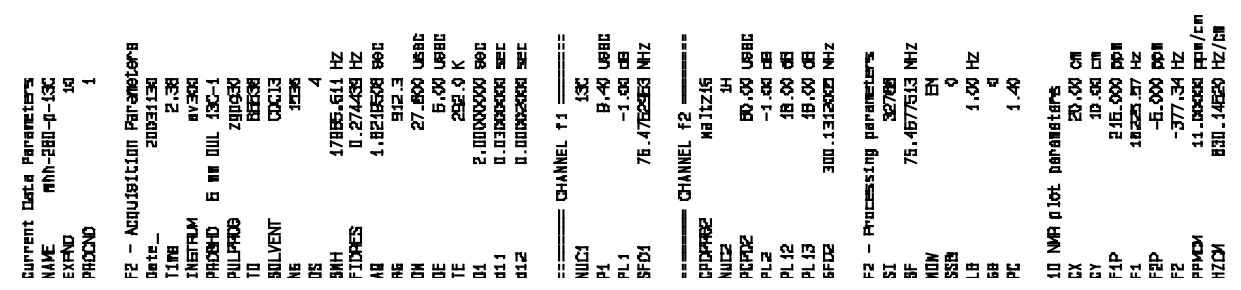

$000^{\circ} 0$
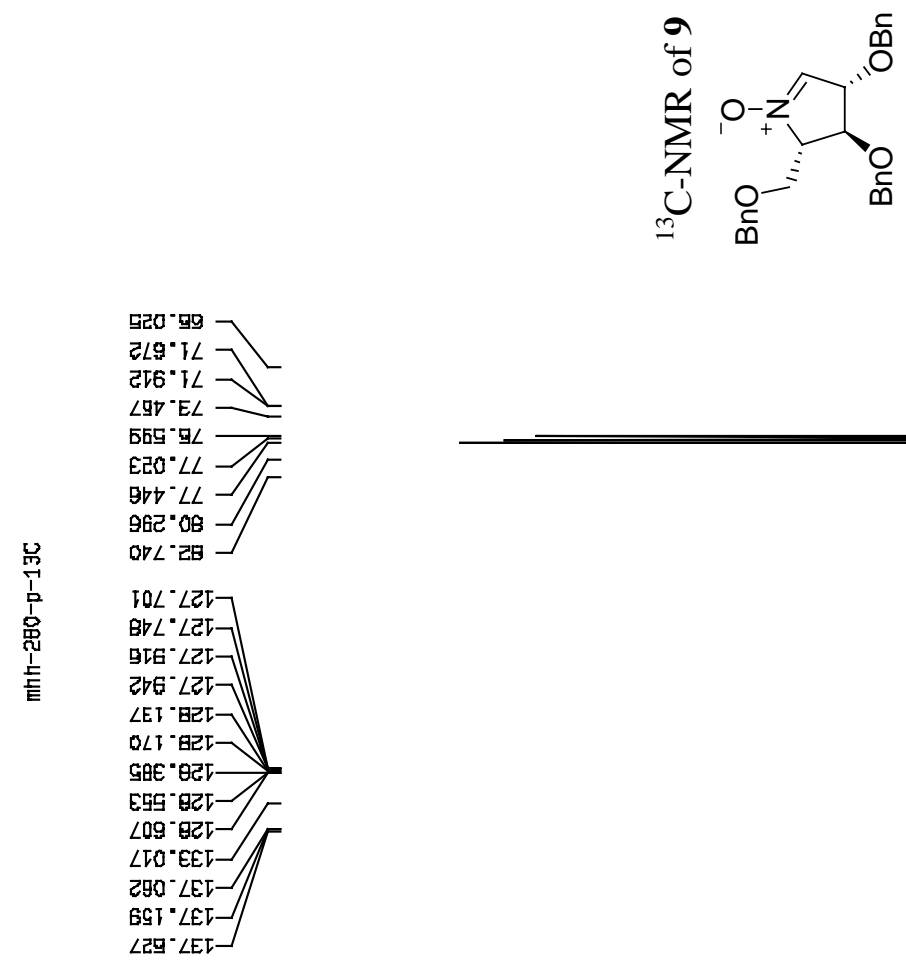

$\frac{\theta}{\text { s่ }}$

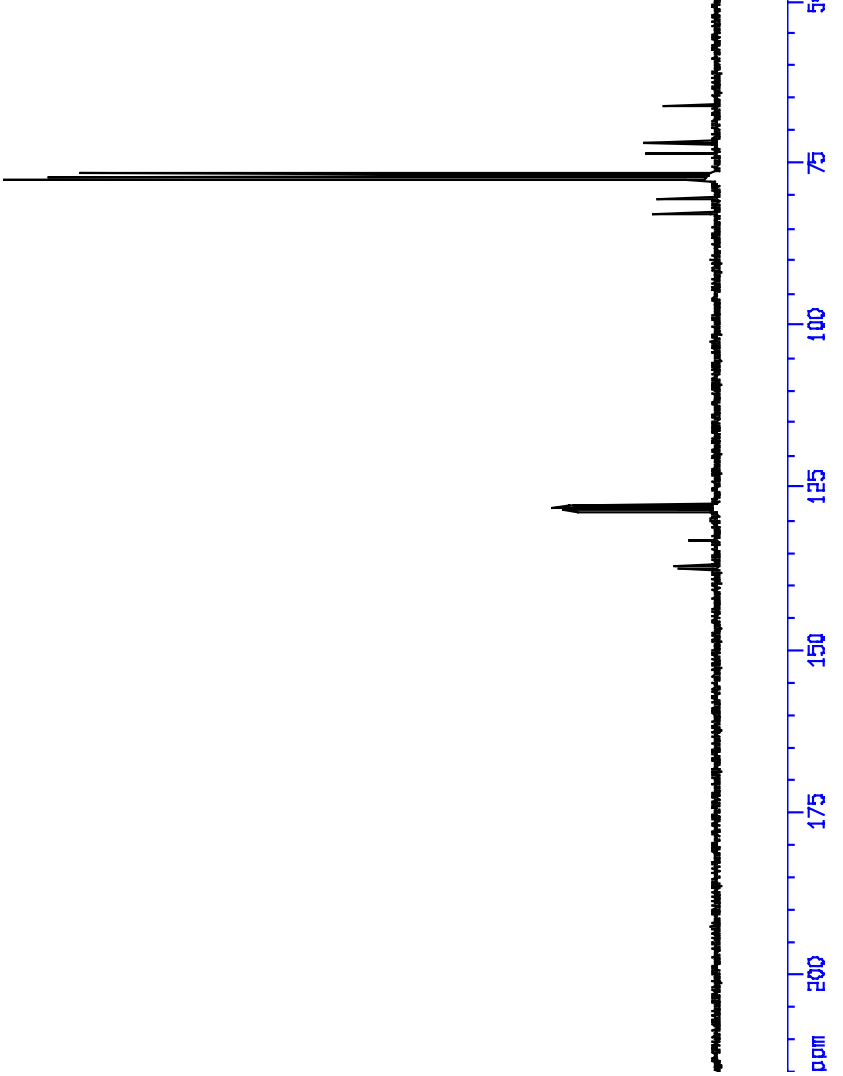



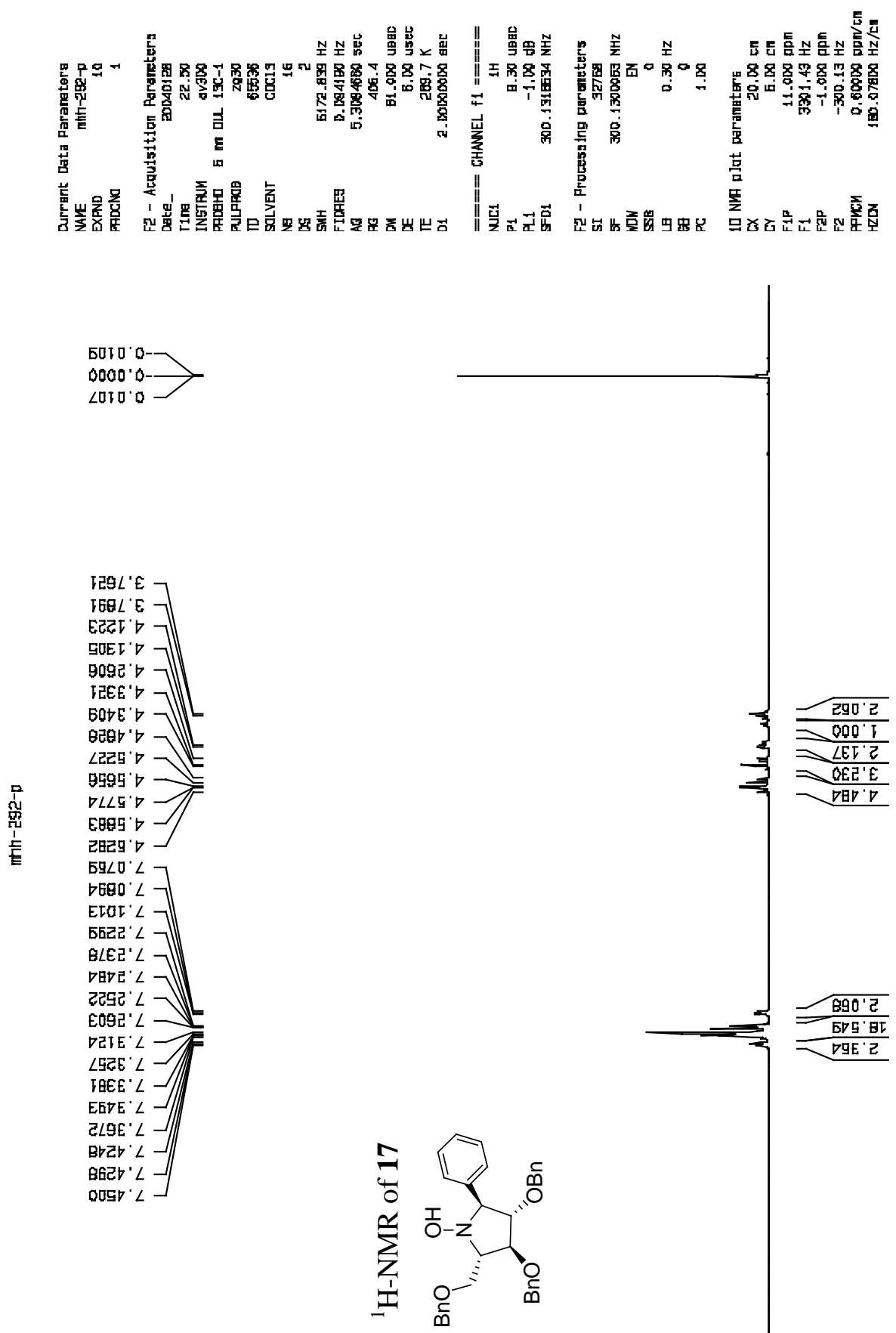

แdd

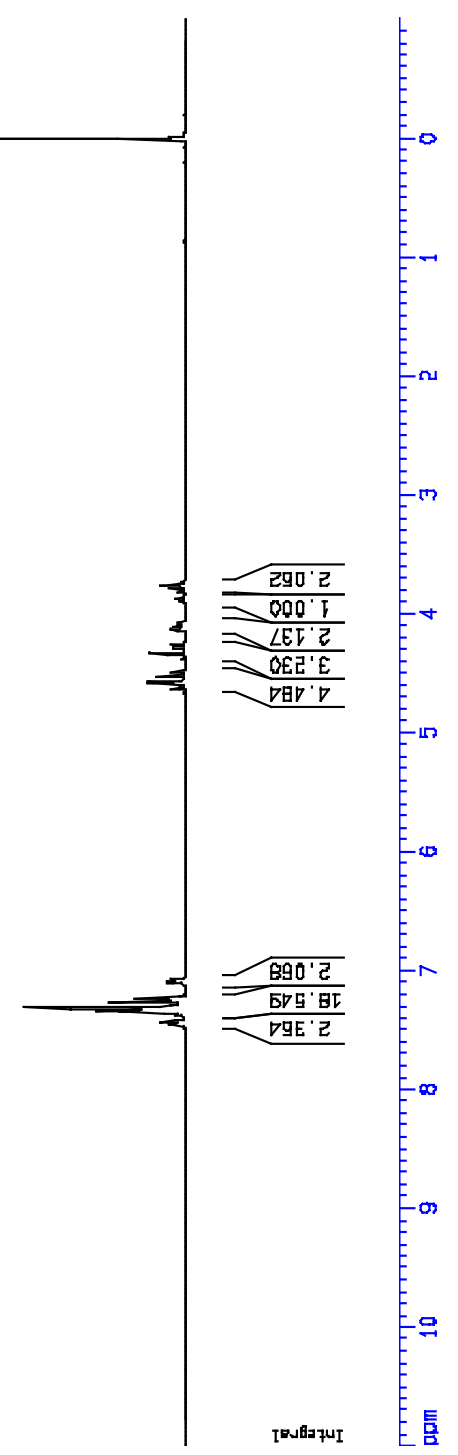

๙ั 

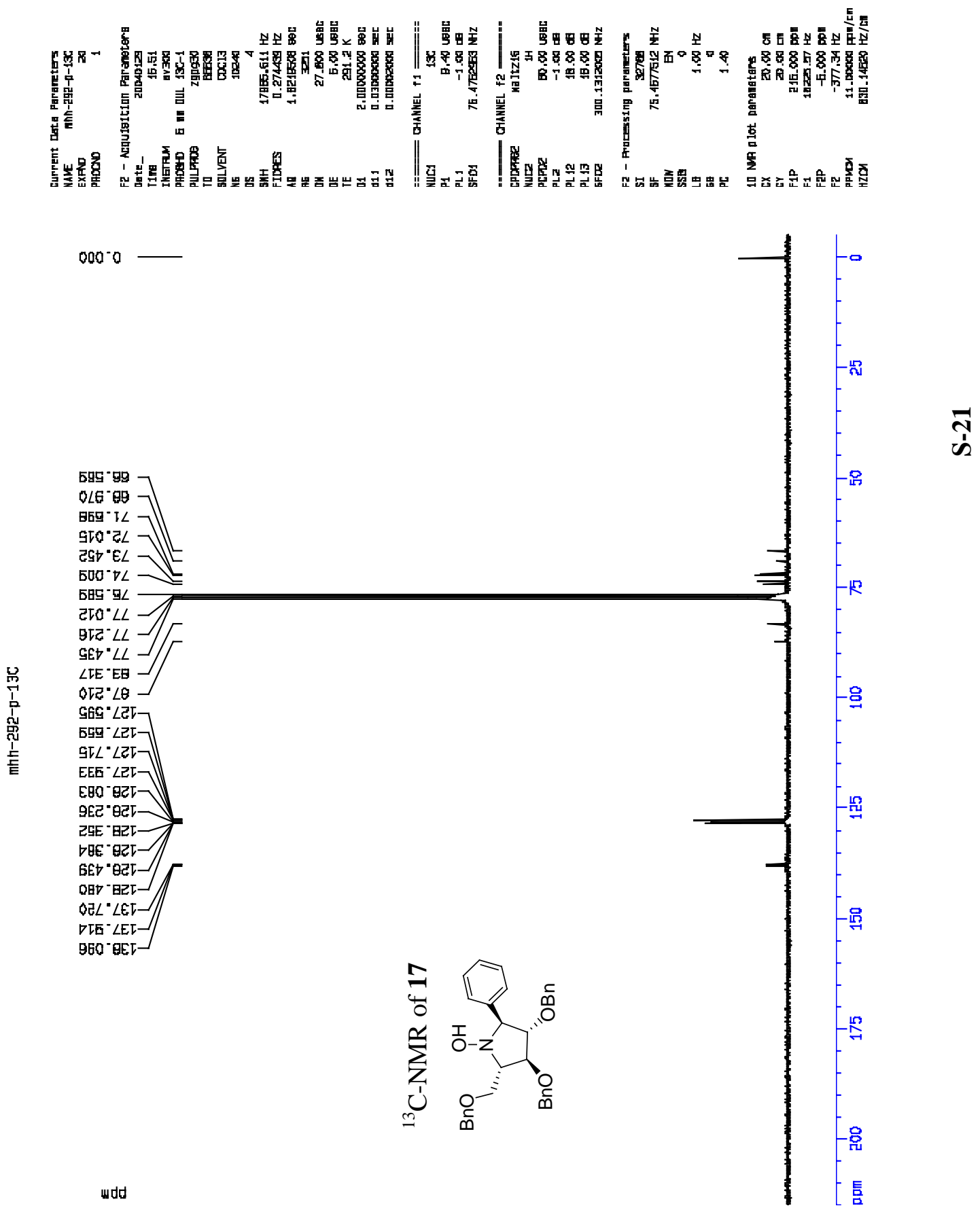

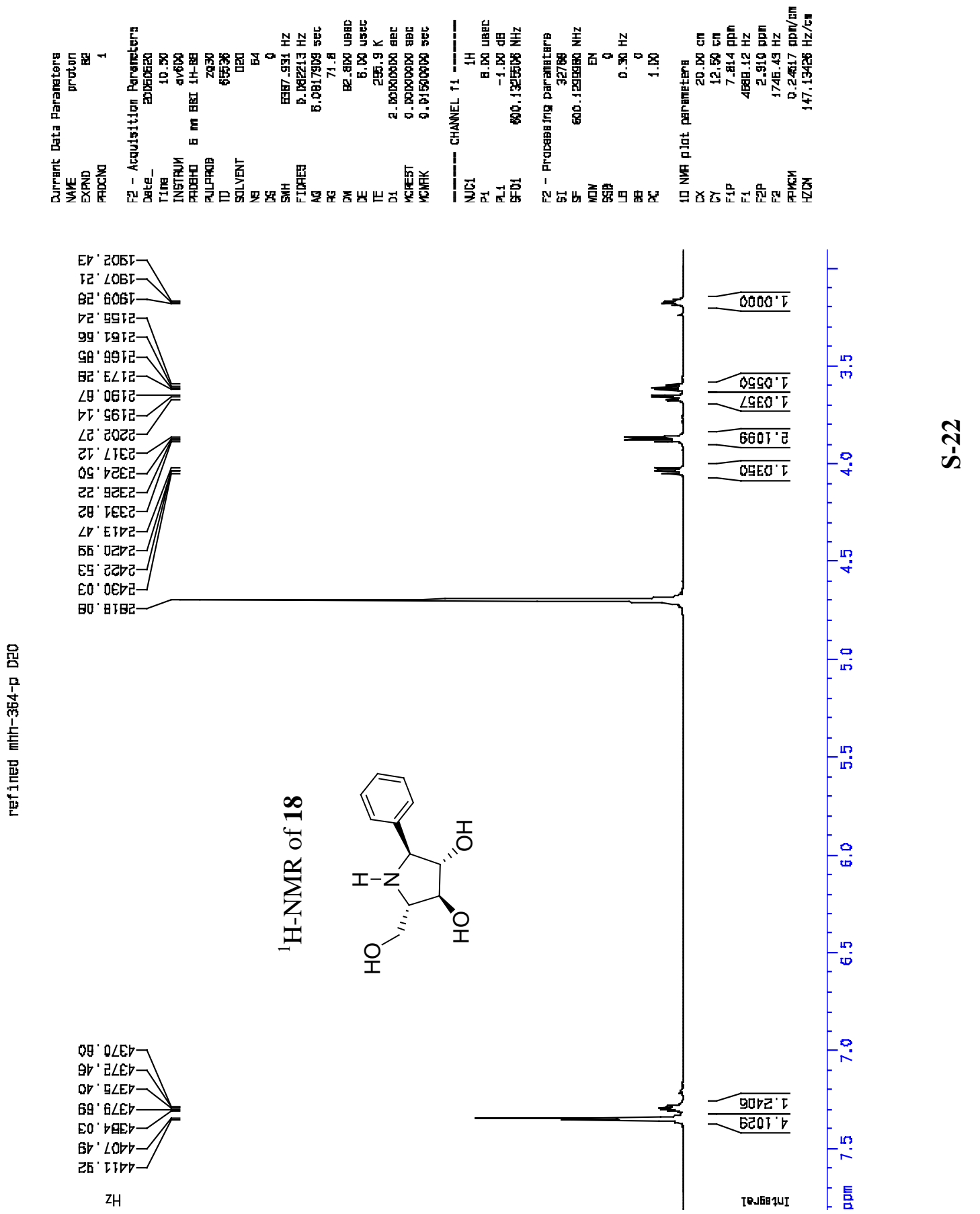

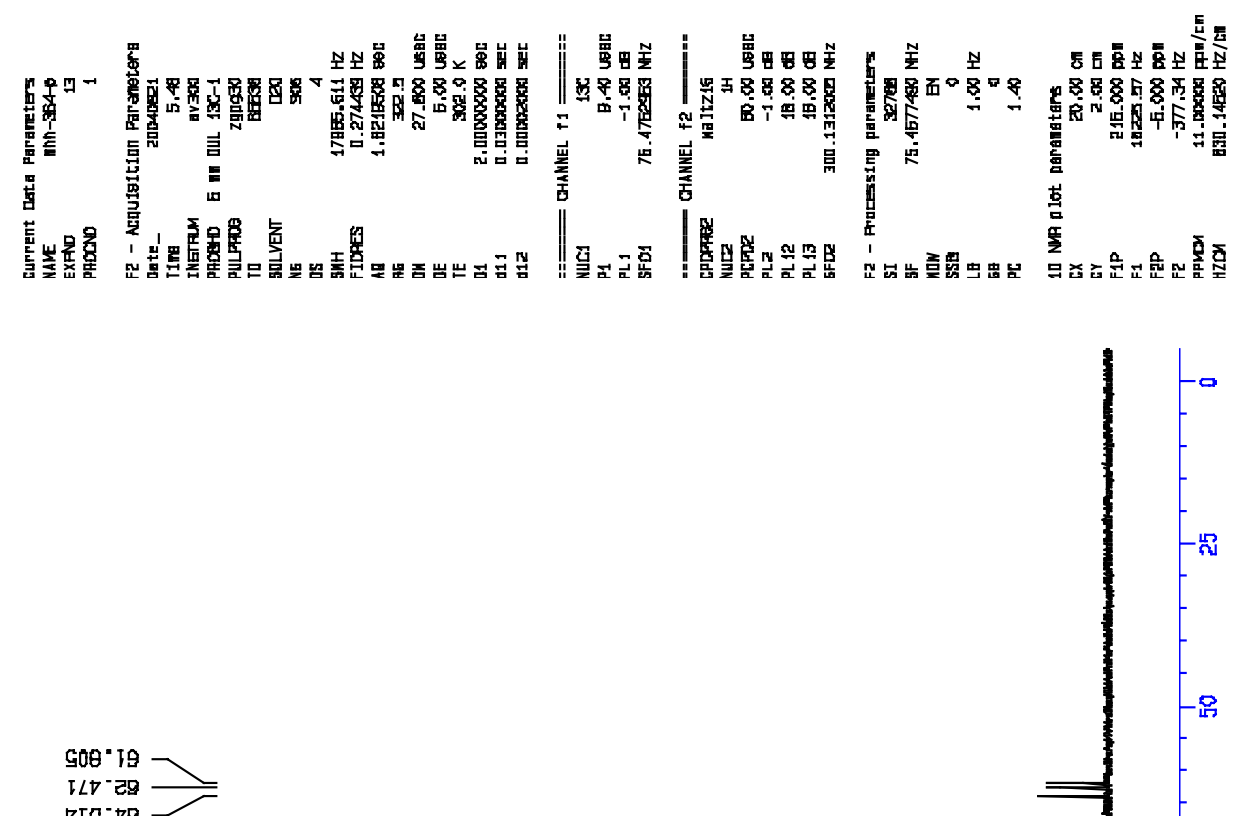

Bटc " $\angle L$

$\checkmark L C^{\circ} Z B$

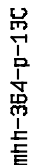
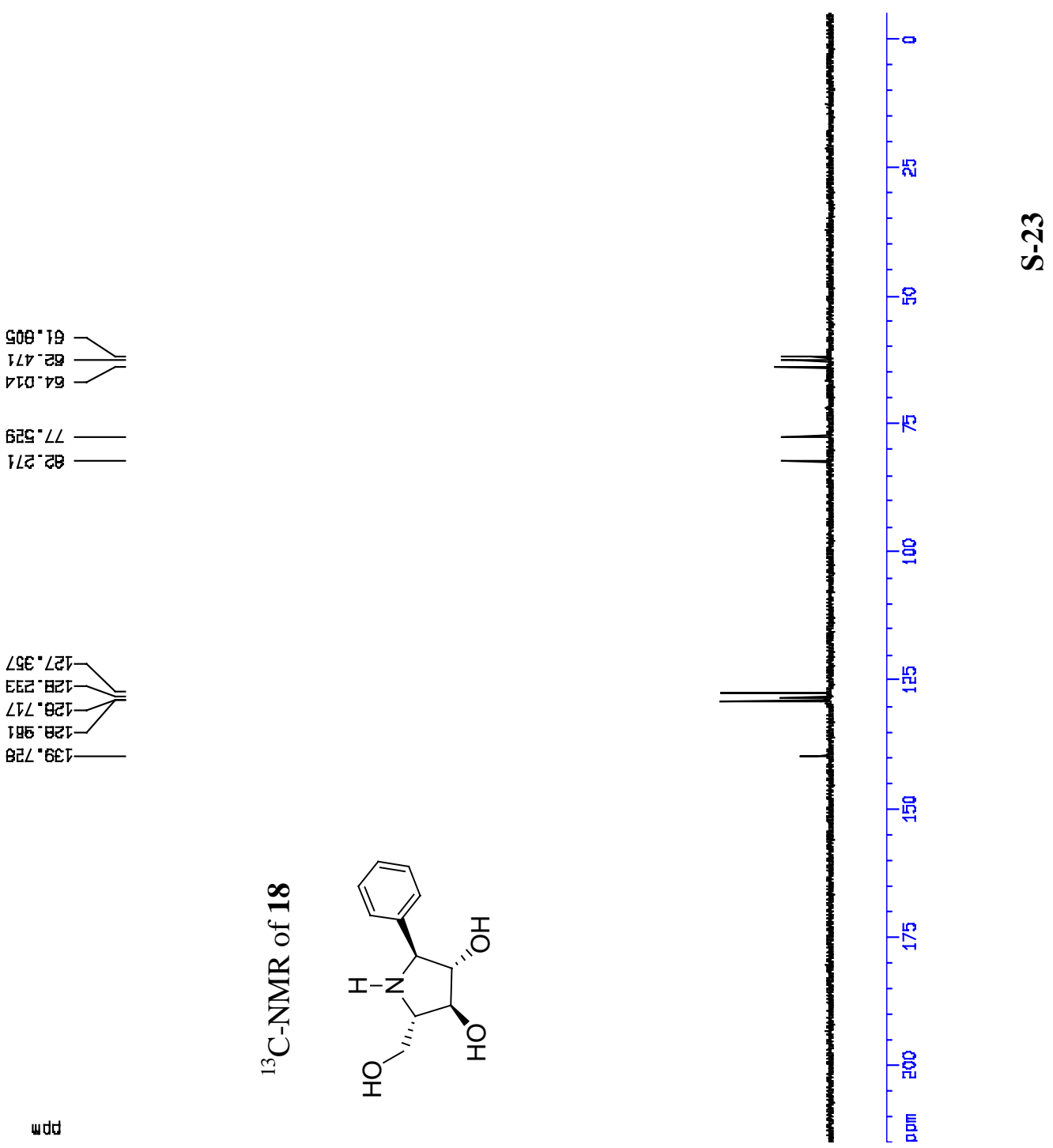


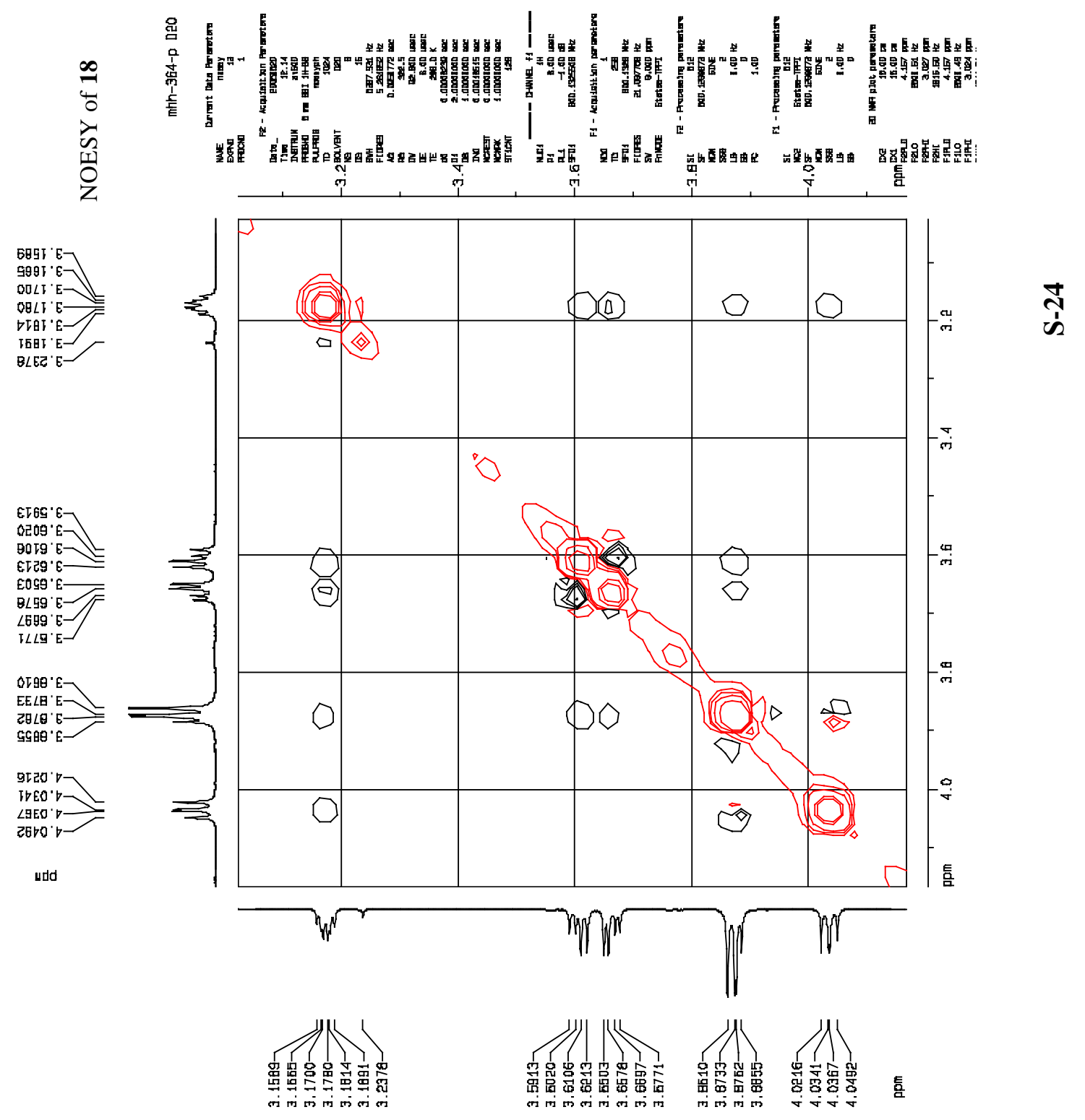




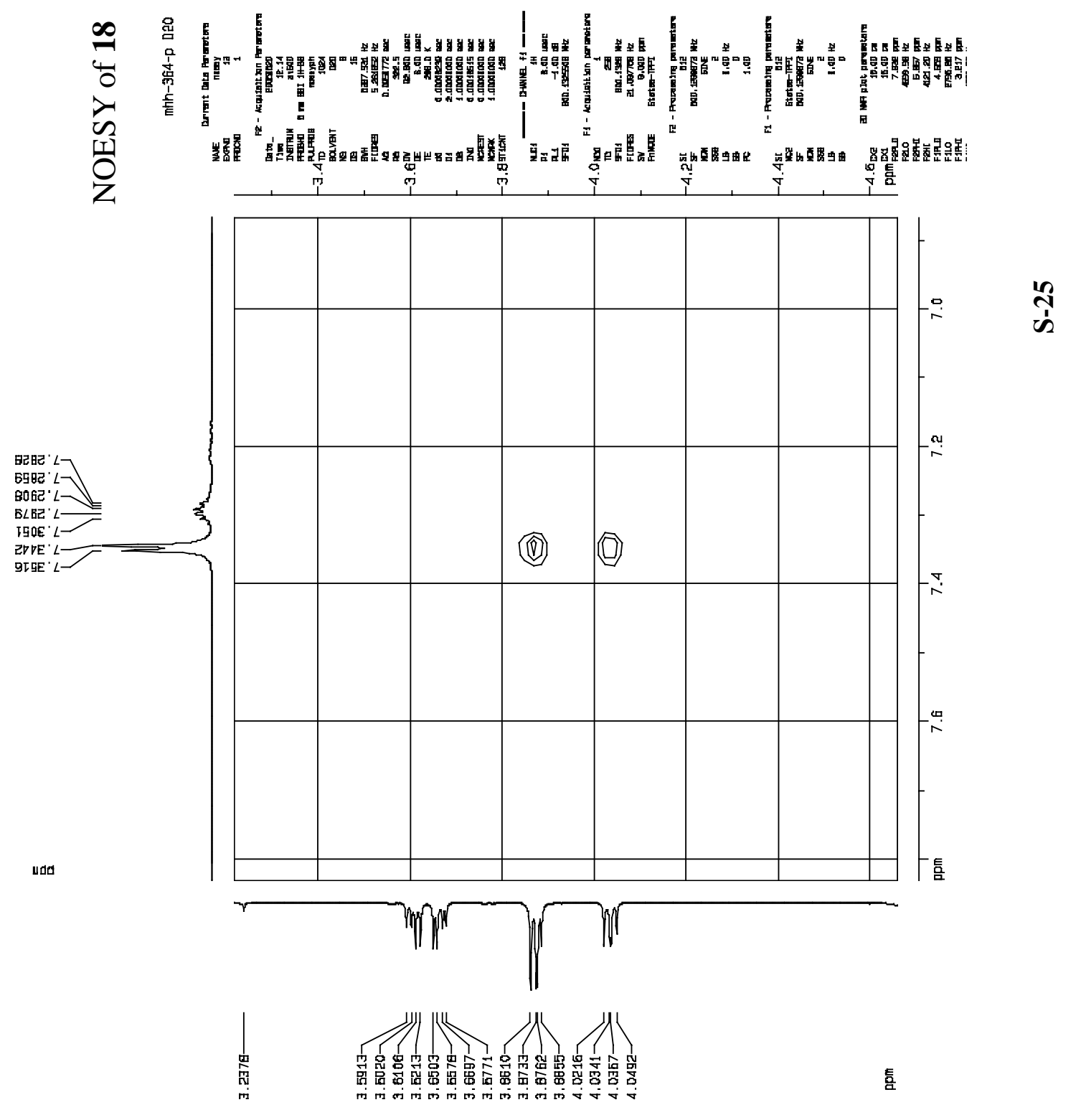



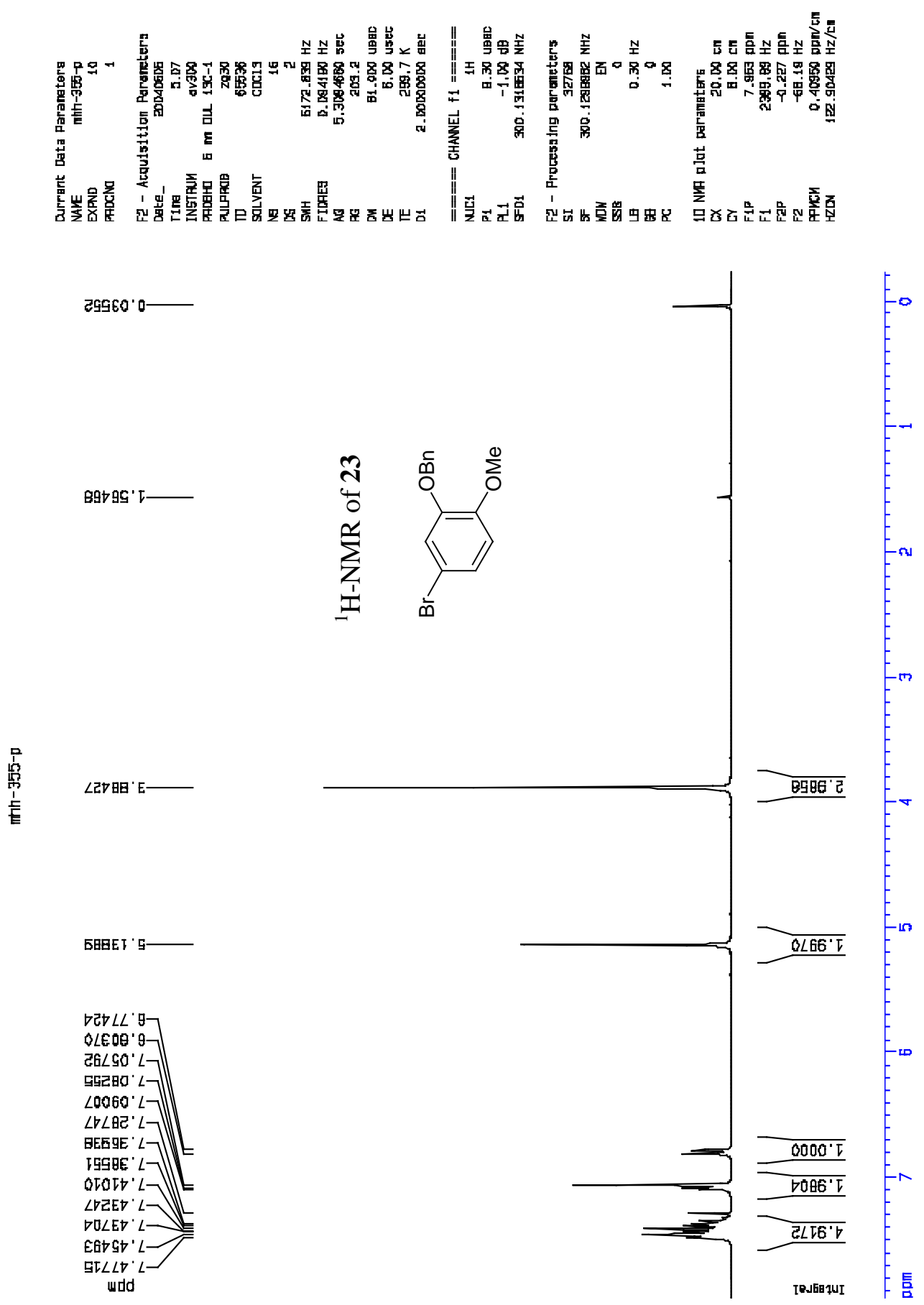

\% 


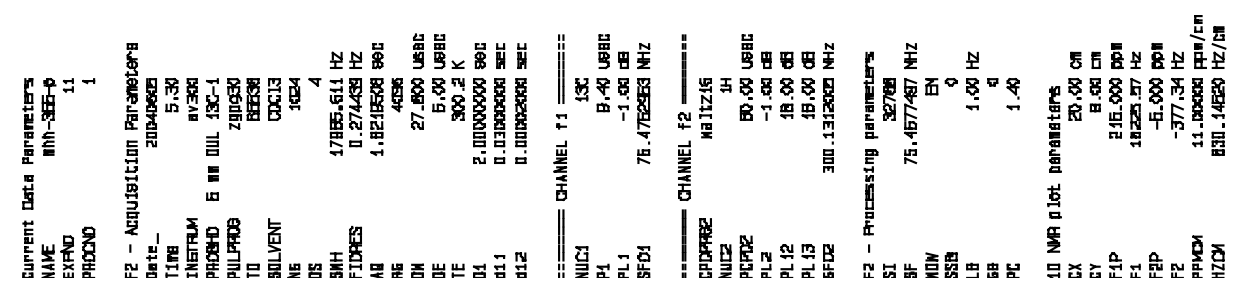

$000^{\circ}$

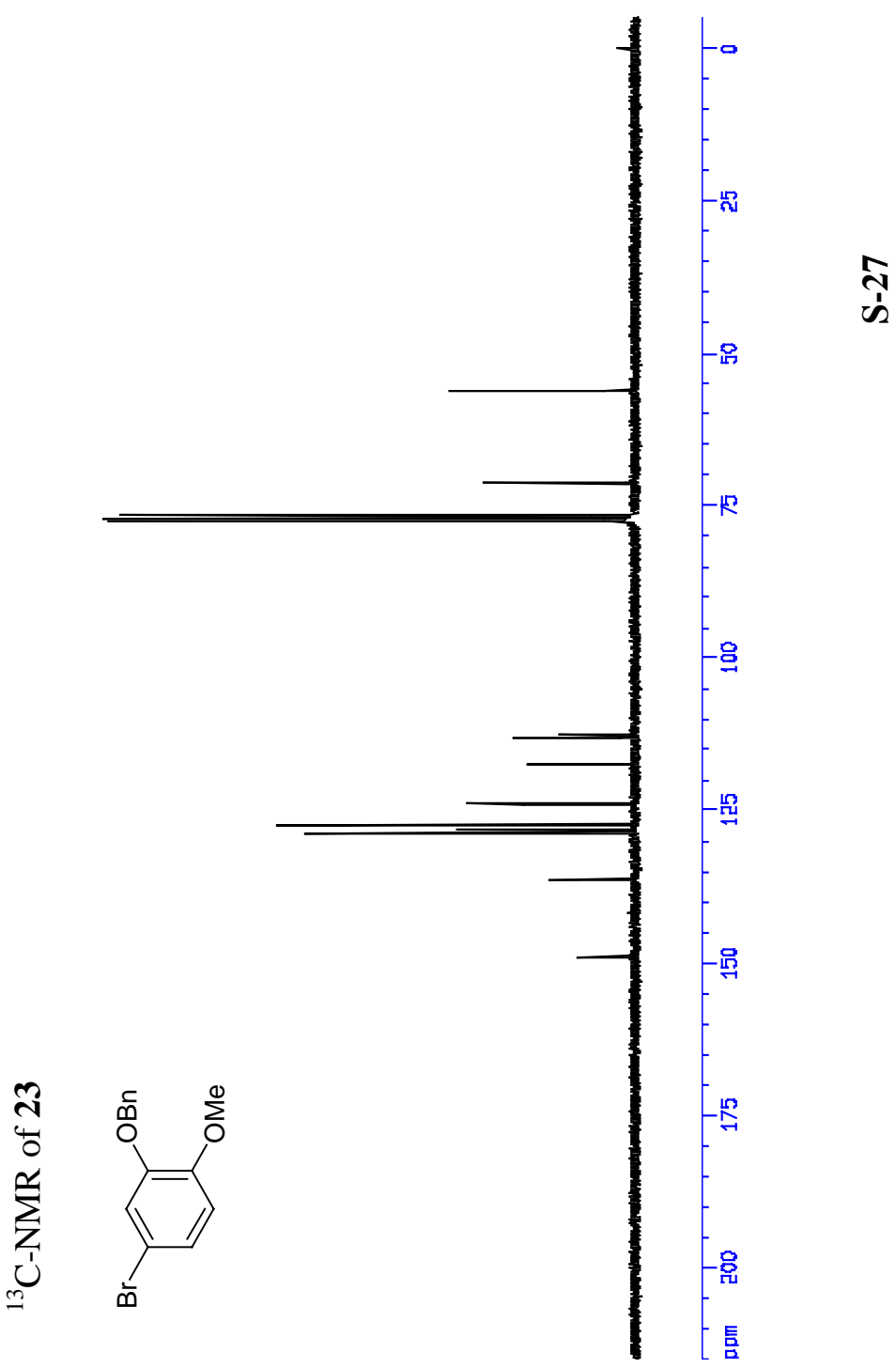

$I L F \cdot 9 G$

$5 b^{\circ} \mathrm{T} L$

Б65 ' $9 \angle>$

$2200^{\circ} \angle L$

5bt $L L$

吕

$\angle G G{ }^{\circ} \mathrm{CLT}$

GET EIT

$O D E-\angle L$

$900^{\circ} \forall 25$

BEE' $\angle 2 I$

टLO

ᄃटg -

$\triangle \nabla \nabla^{\circ}$ gEL

B5E ${ }^{\circ}$ 바

$590^{\circ} 605$ 

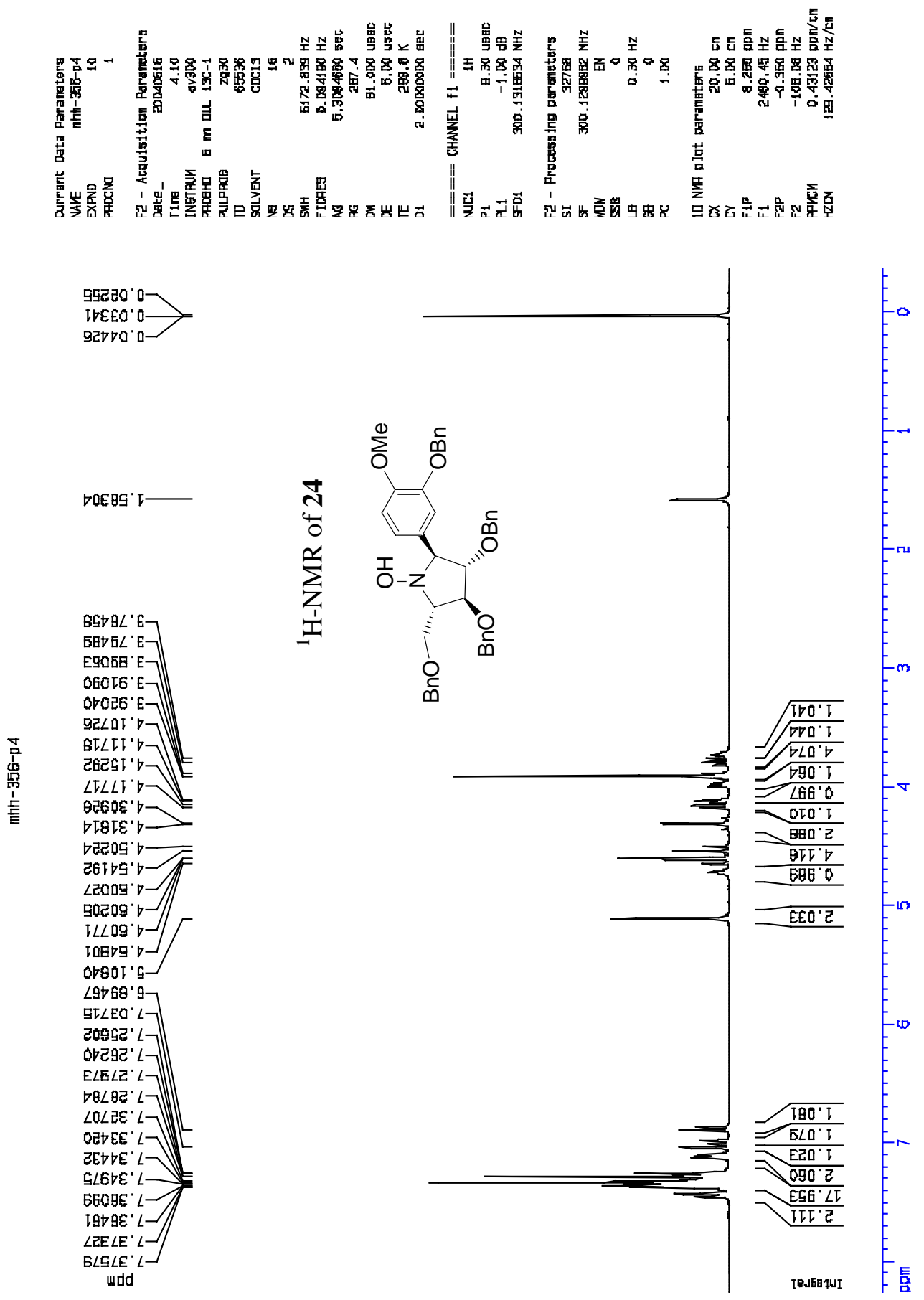

ஸั 

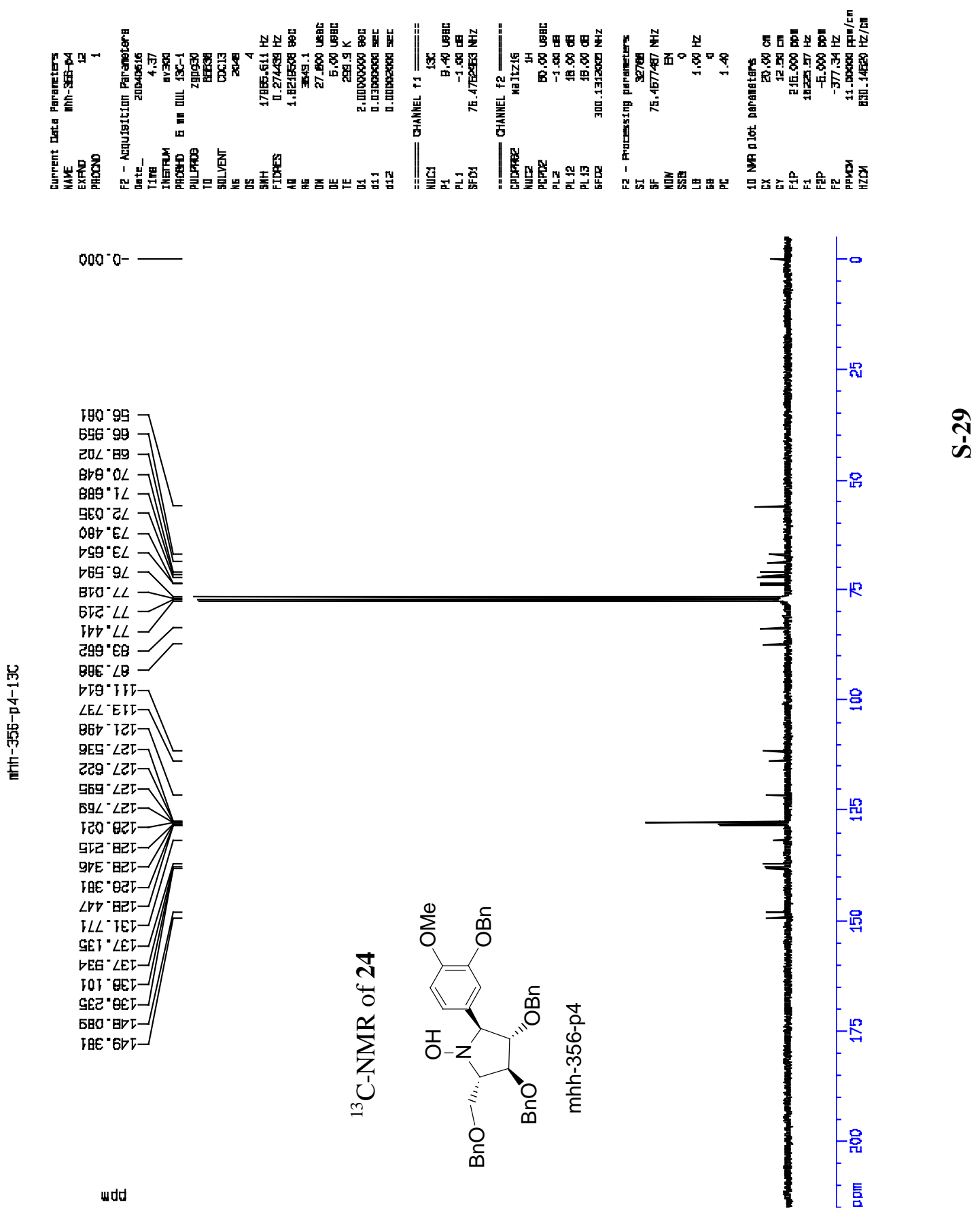

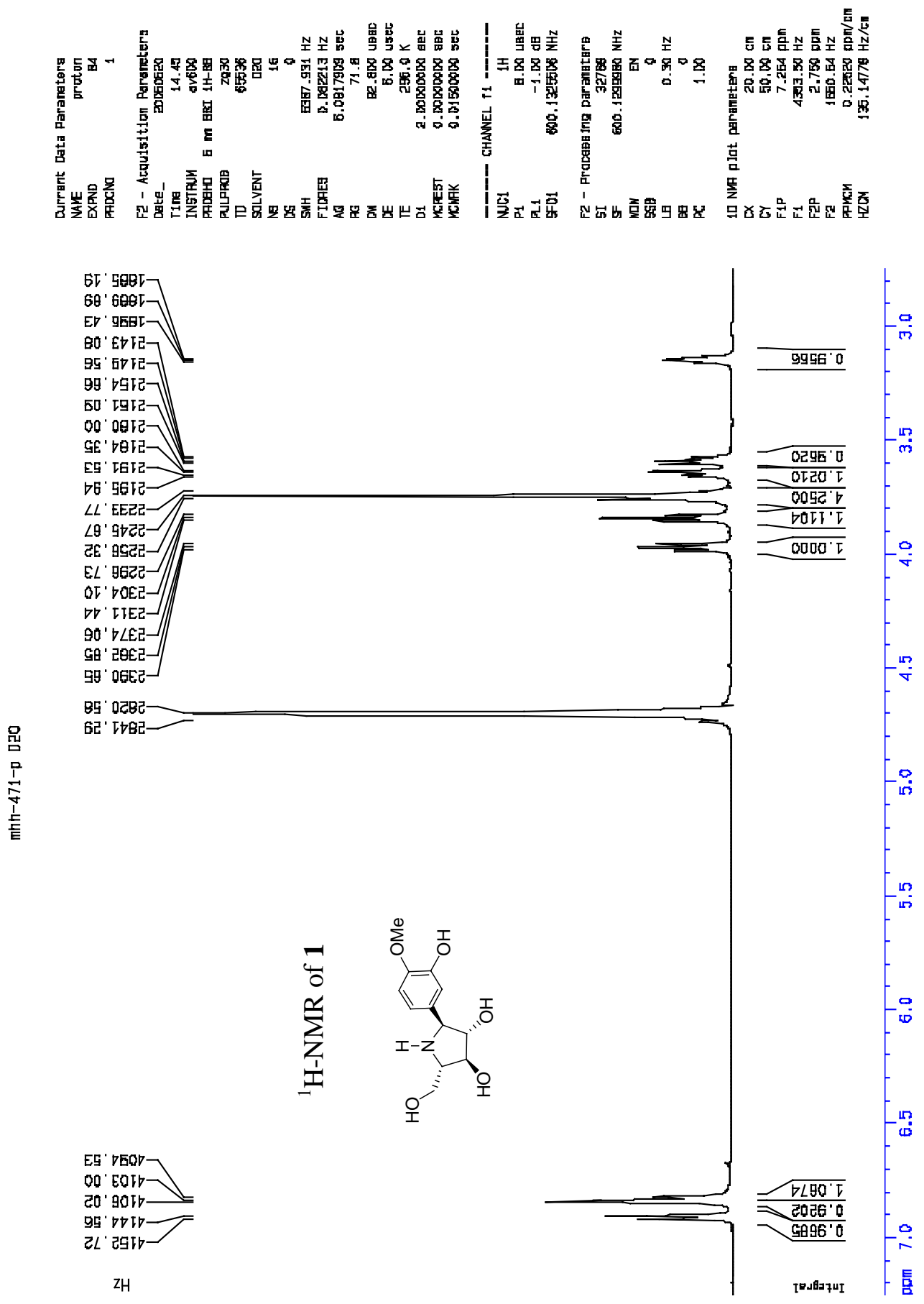

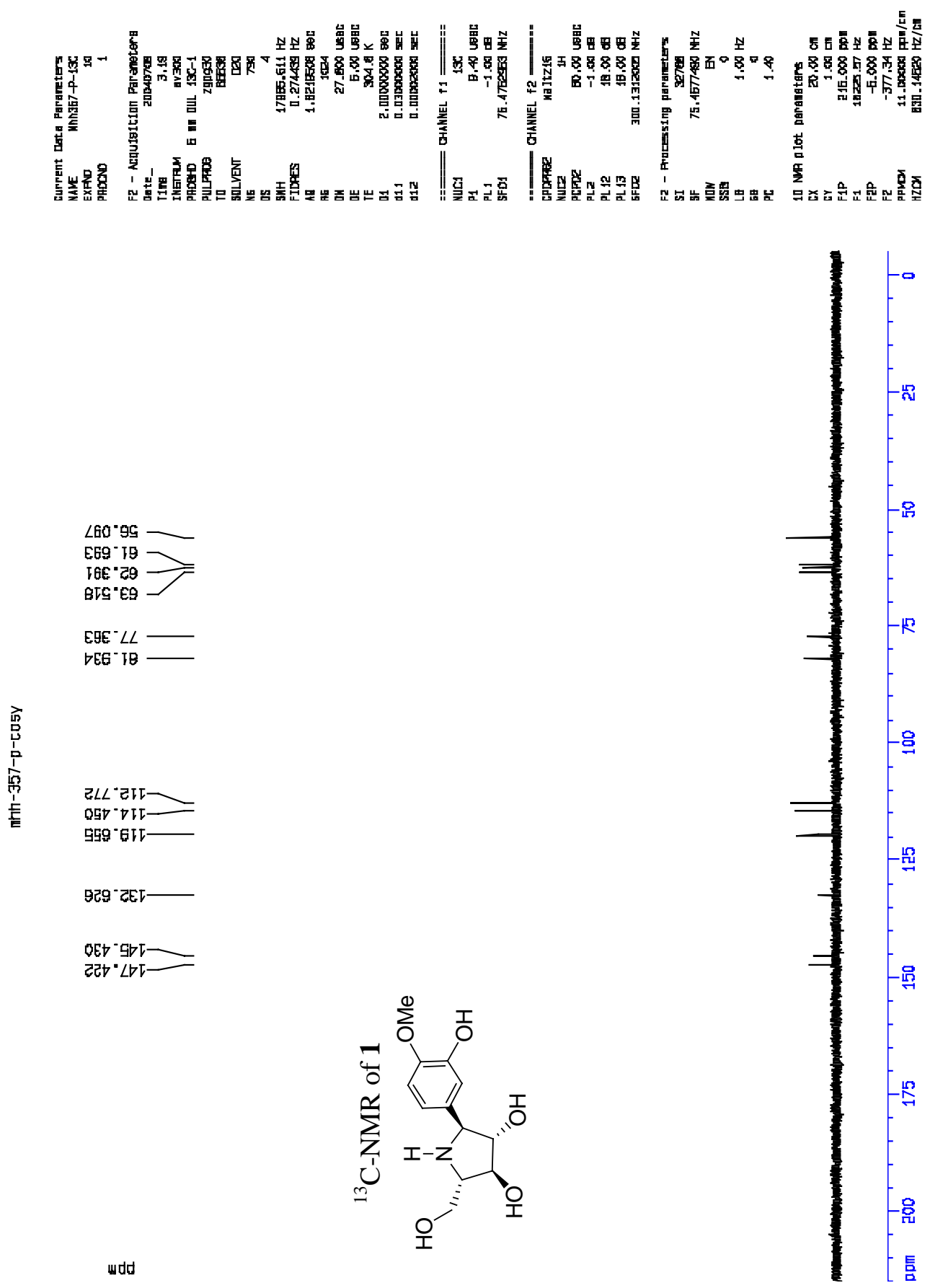

$\overline{3}$ 


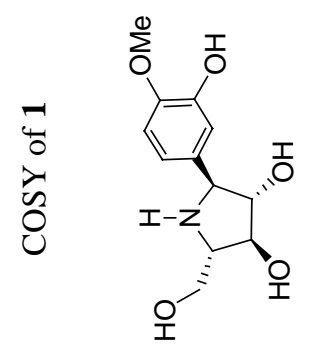

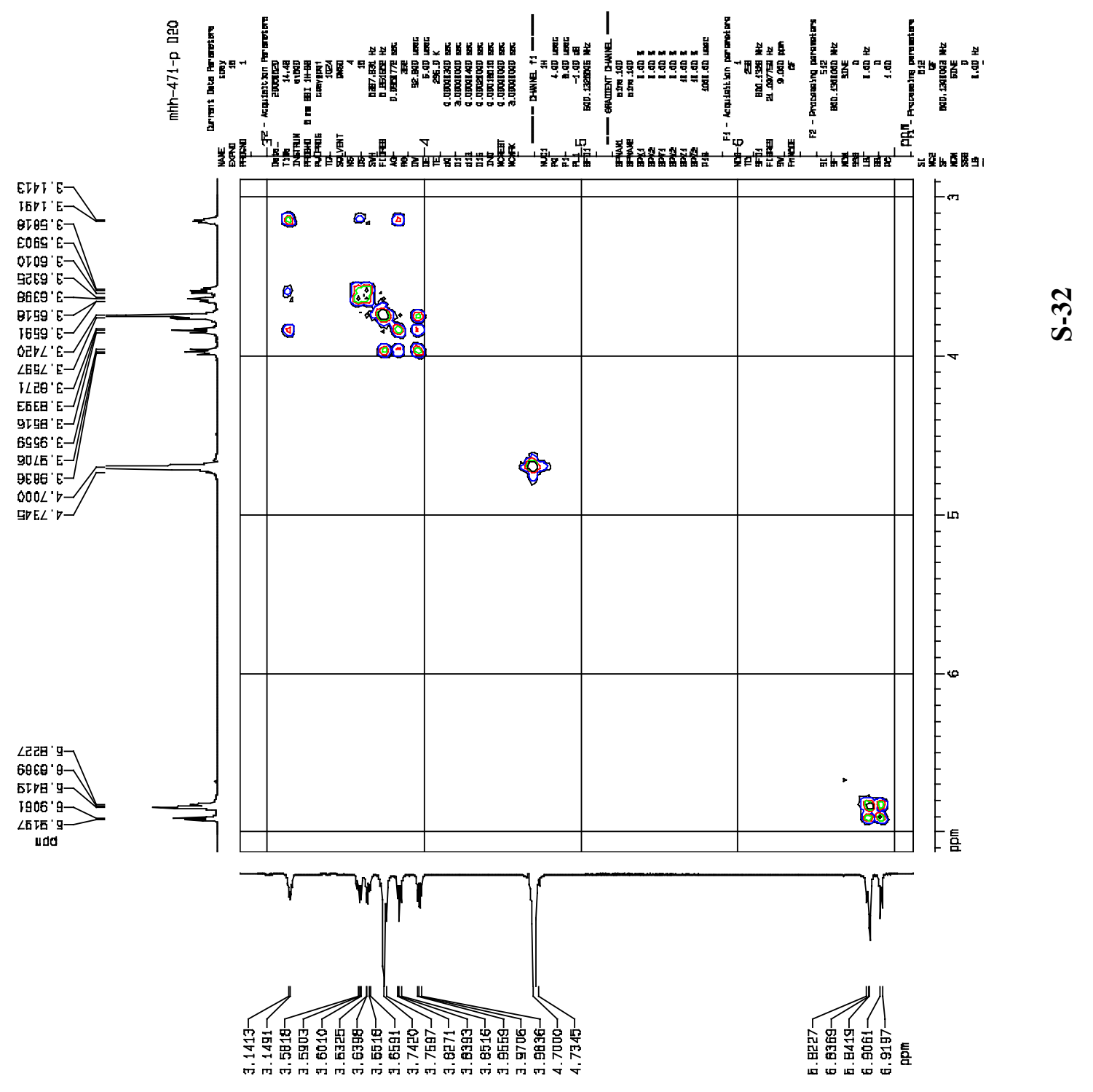



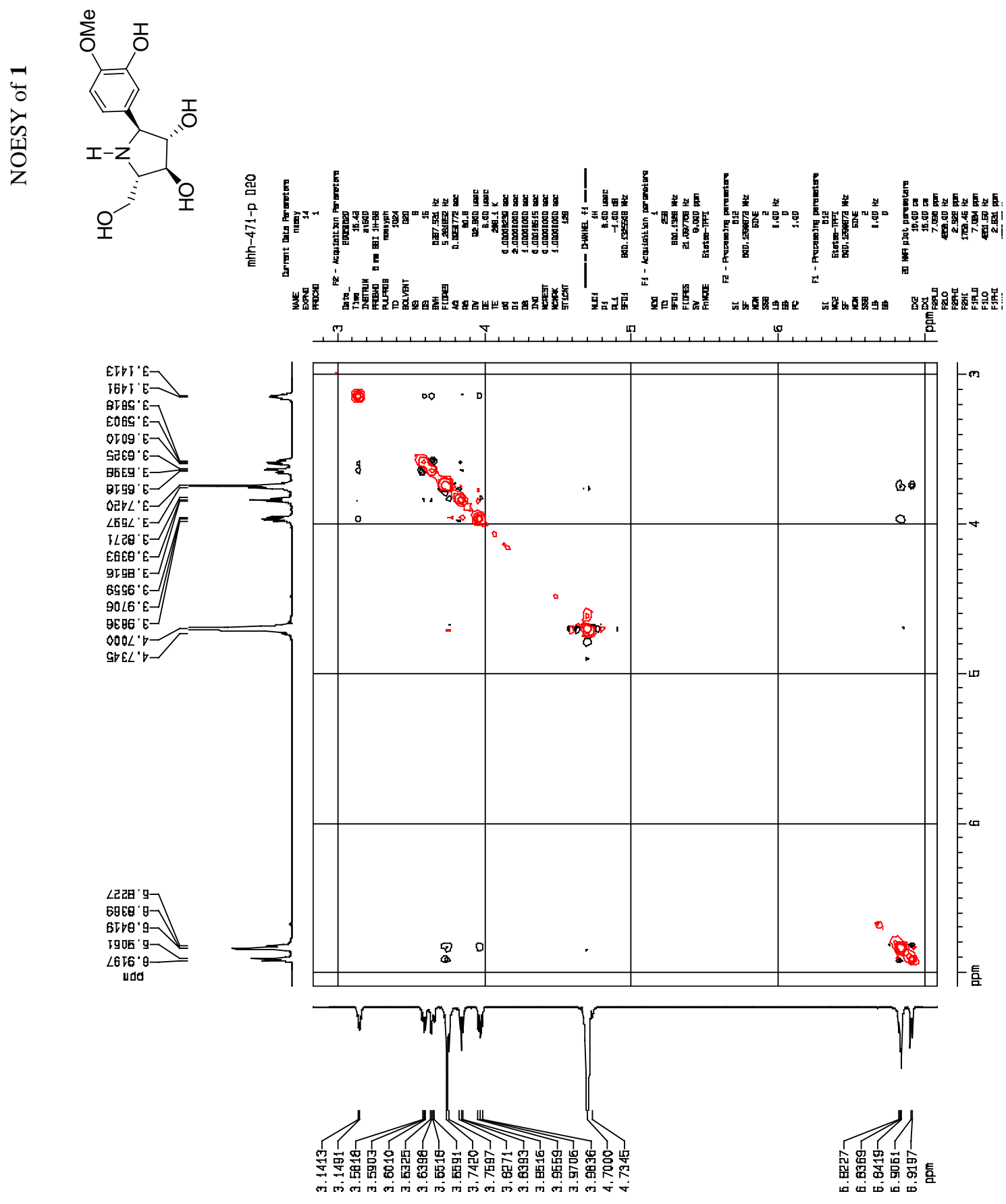

m 


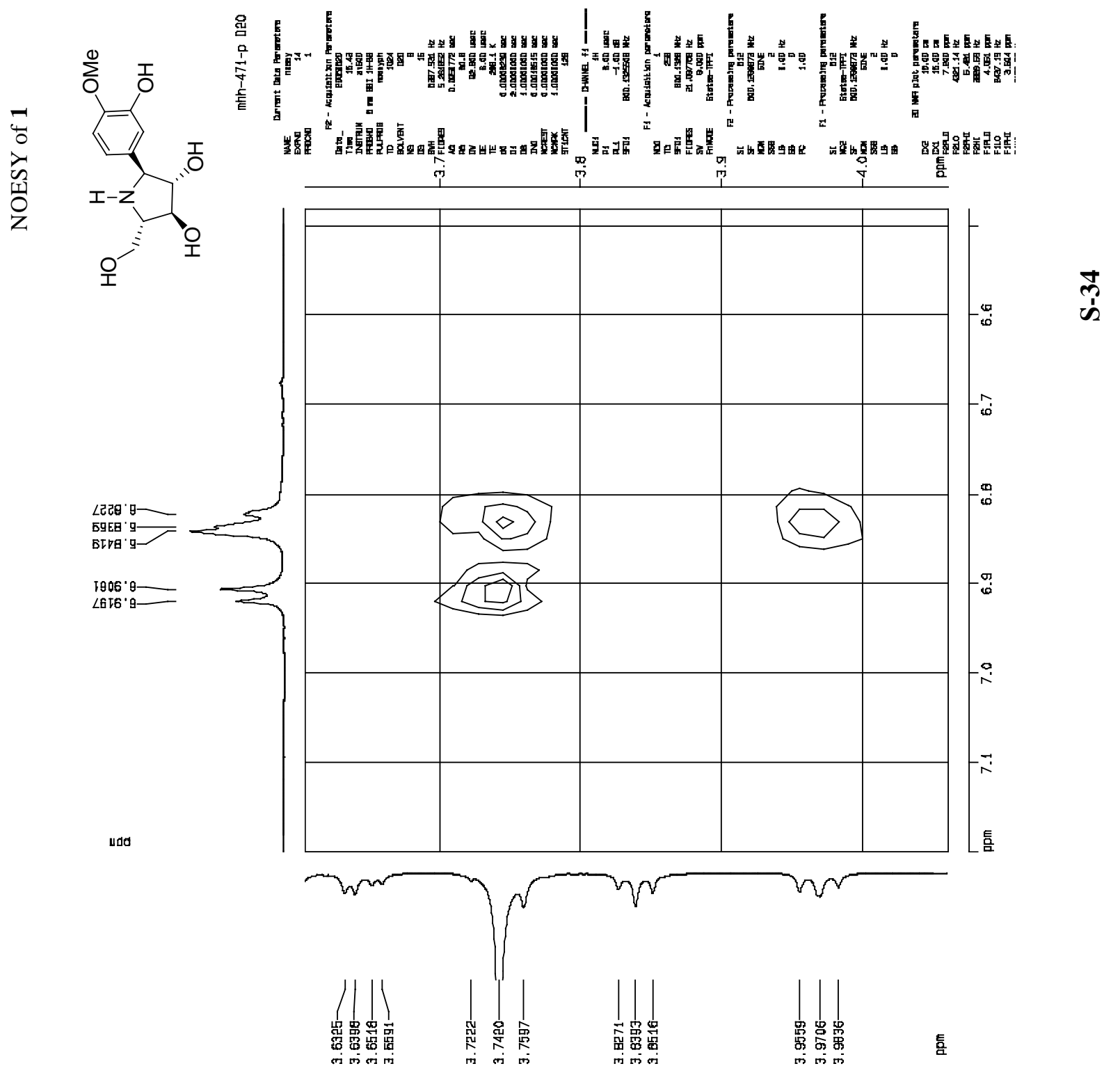



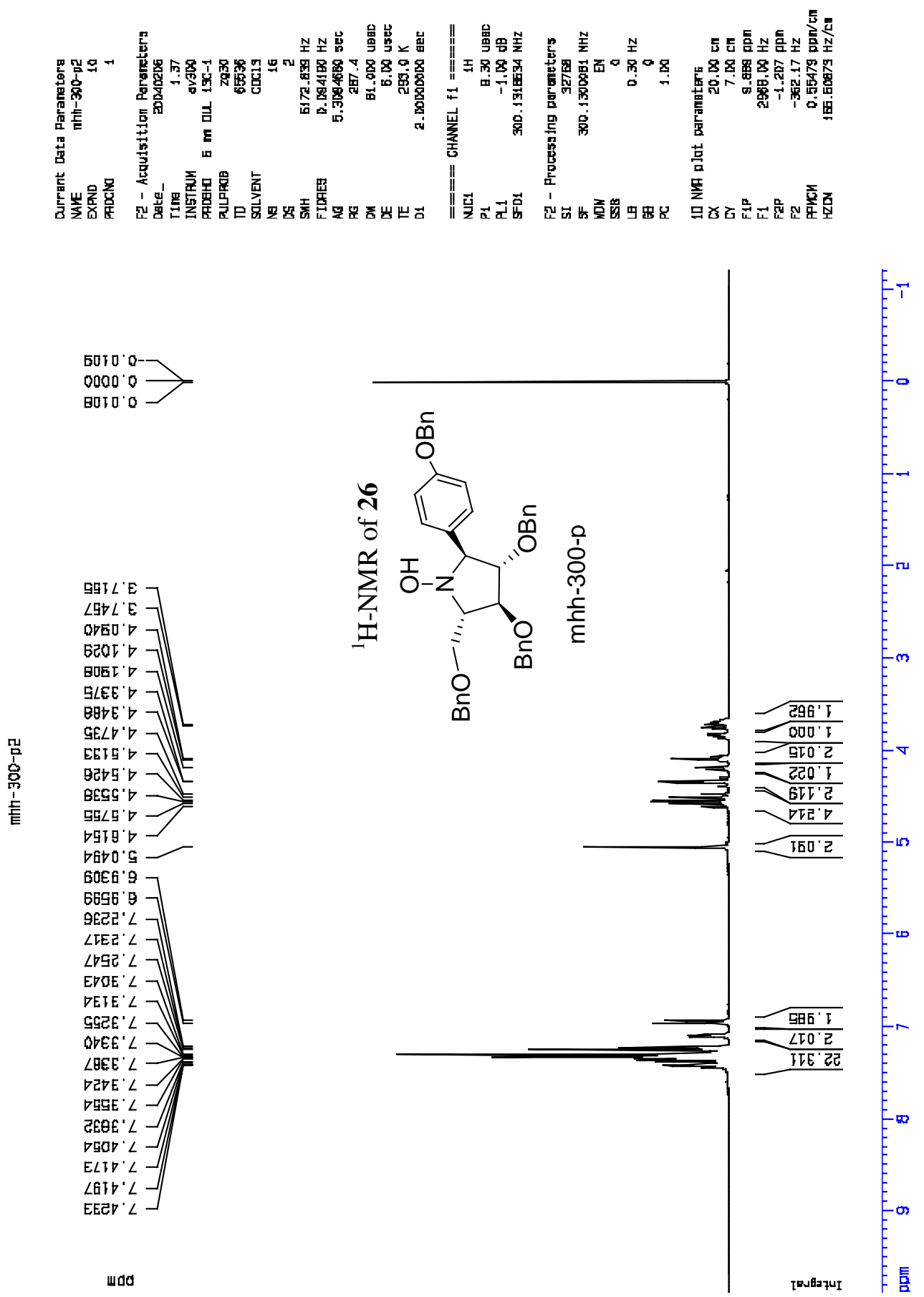

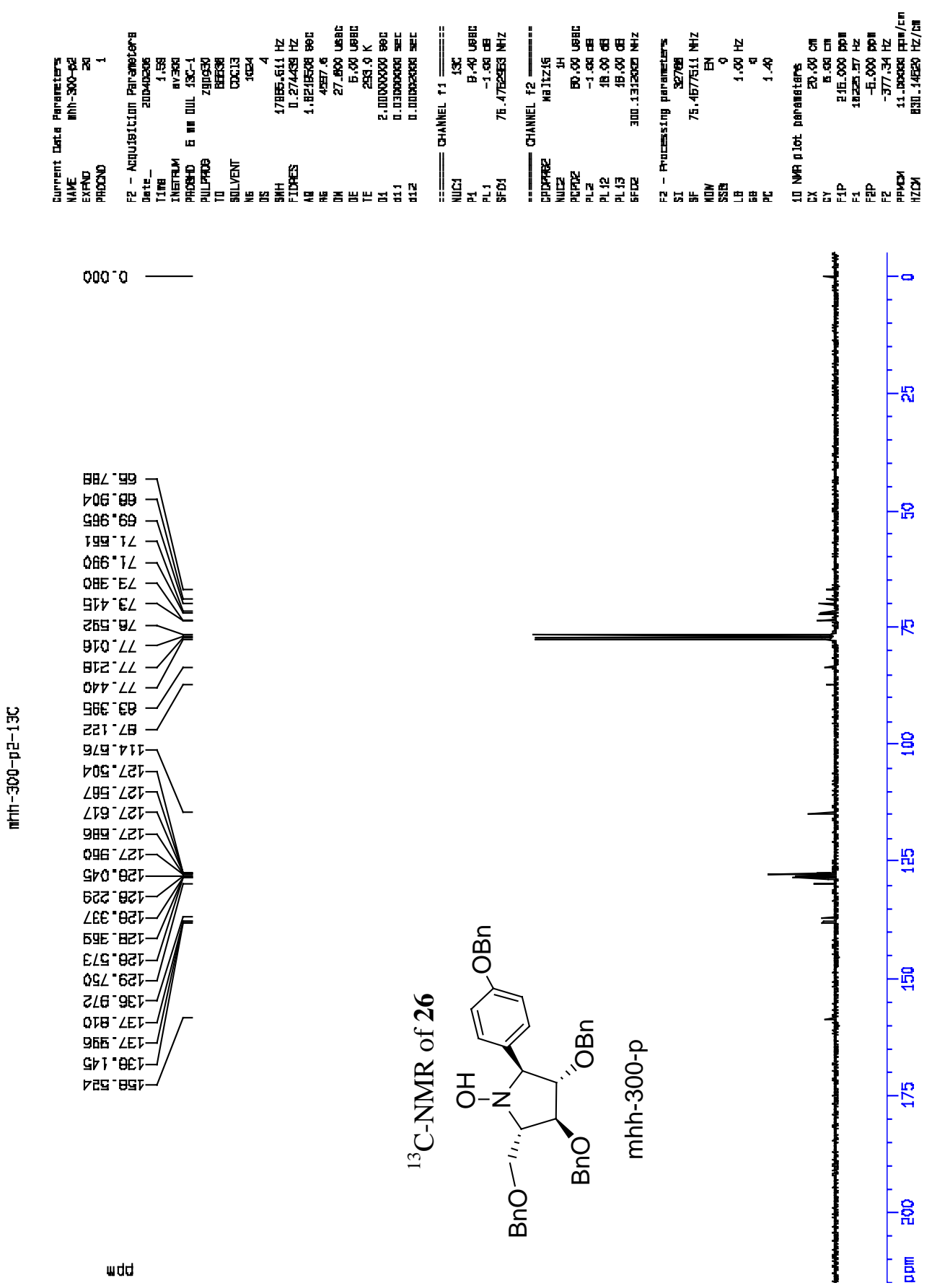

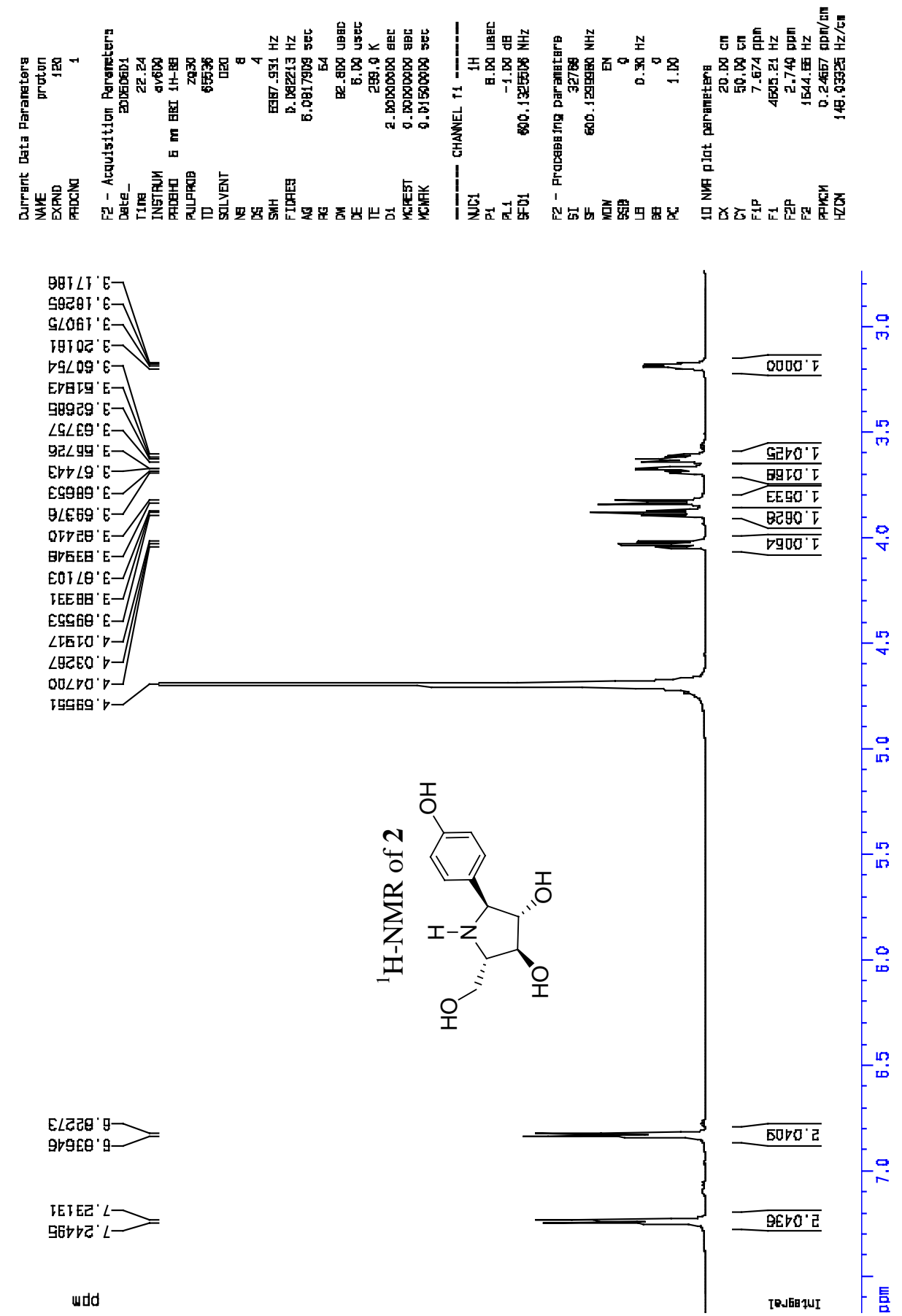

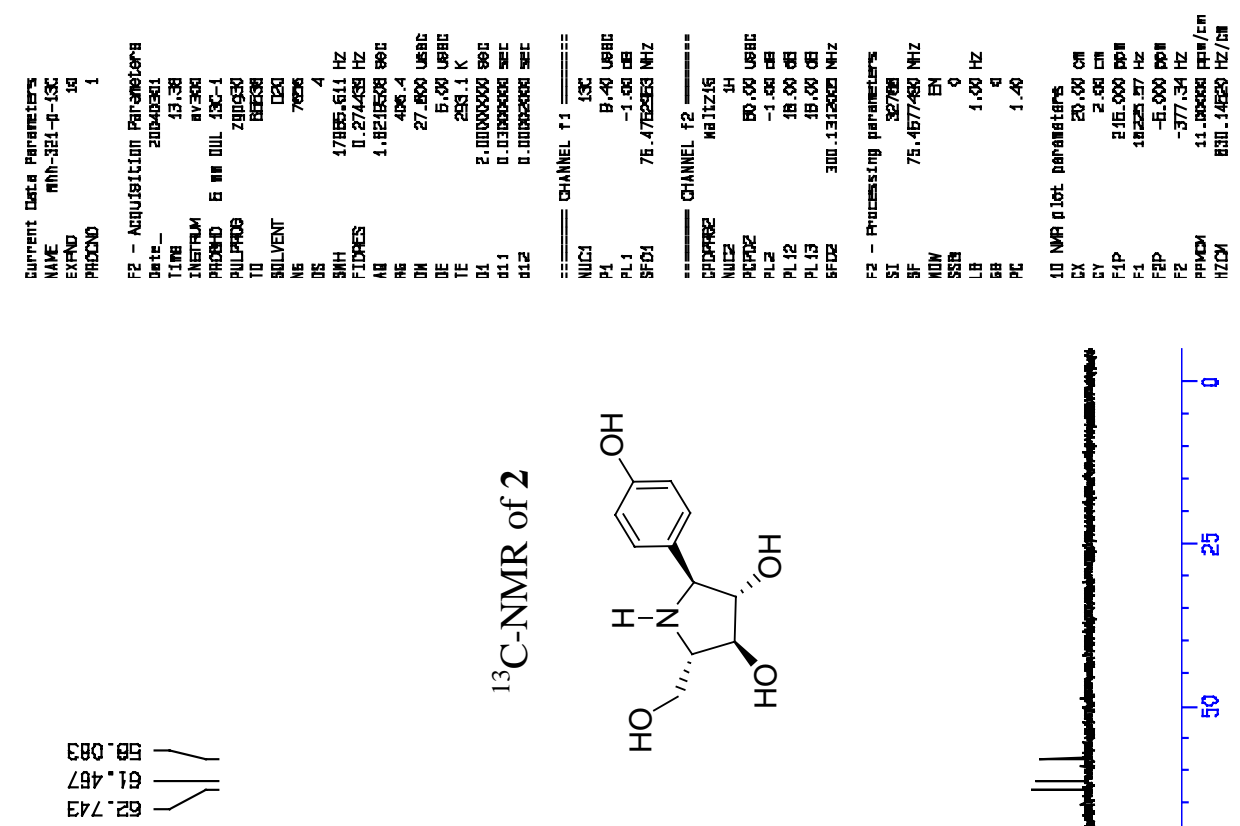

IEG 'EL

$290^{\circ} \angle L$

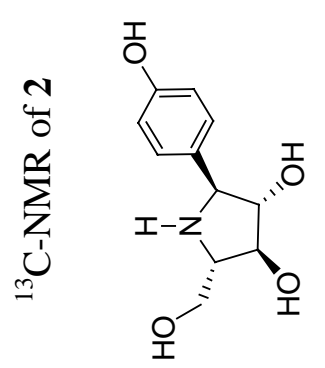

ยนเงเ—

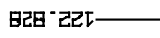

OZT OEГ-

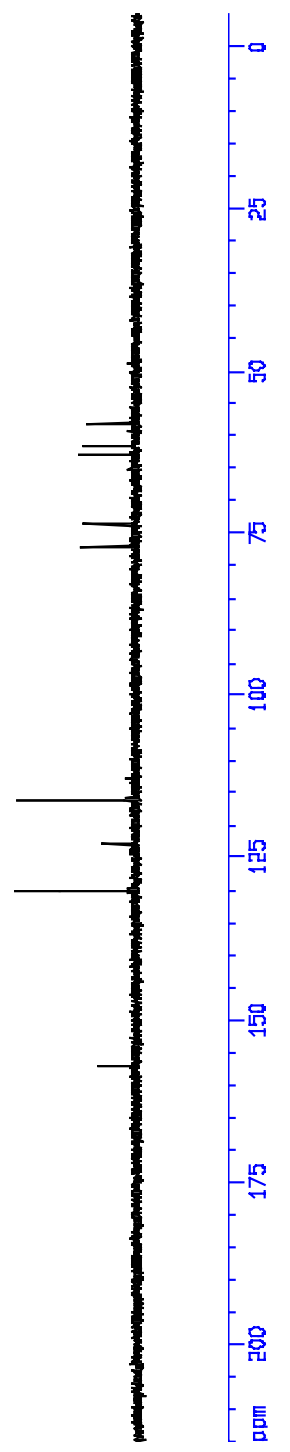


1
0
0
0
0
0
1
1
1
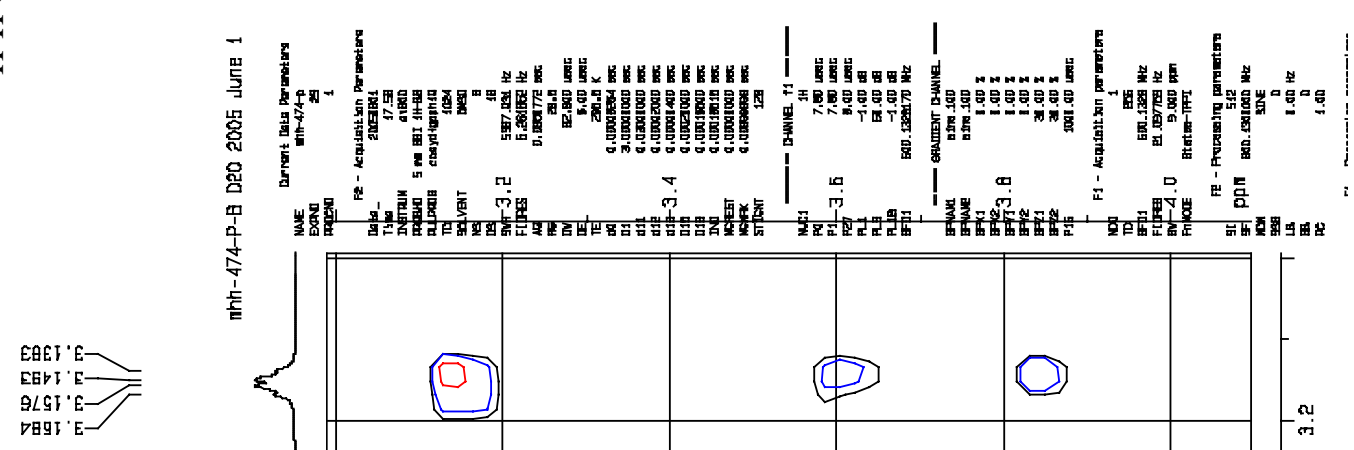

96LS E-

QE $6 S^{\prime} E$

Bbog' $E$ -

EbE E

Gib9 E-

DEGg E-

O

$968 \angle \mathrm{E}-$

$5008 \mathrm{E}-$

9LE日 E-

Loge' $\mathrm{E}-$

GL66'E-

O5 $500 \mathrm{D}$

udd

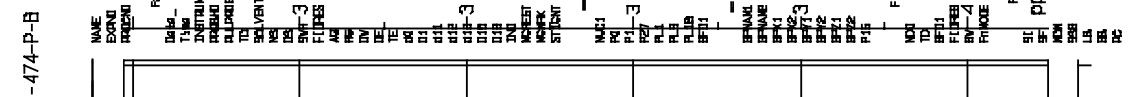

갈
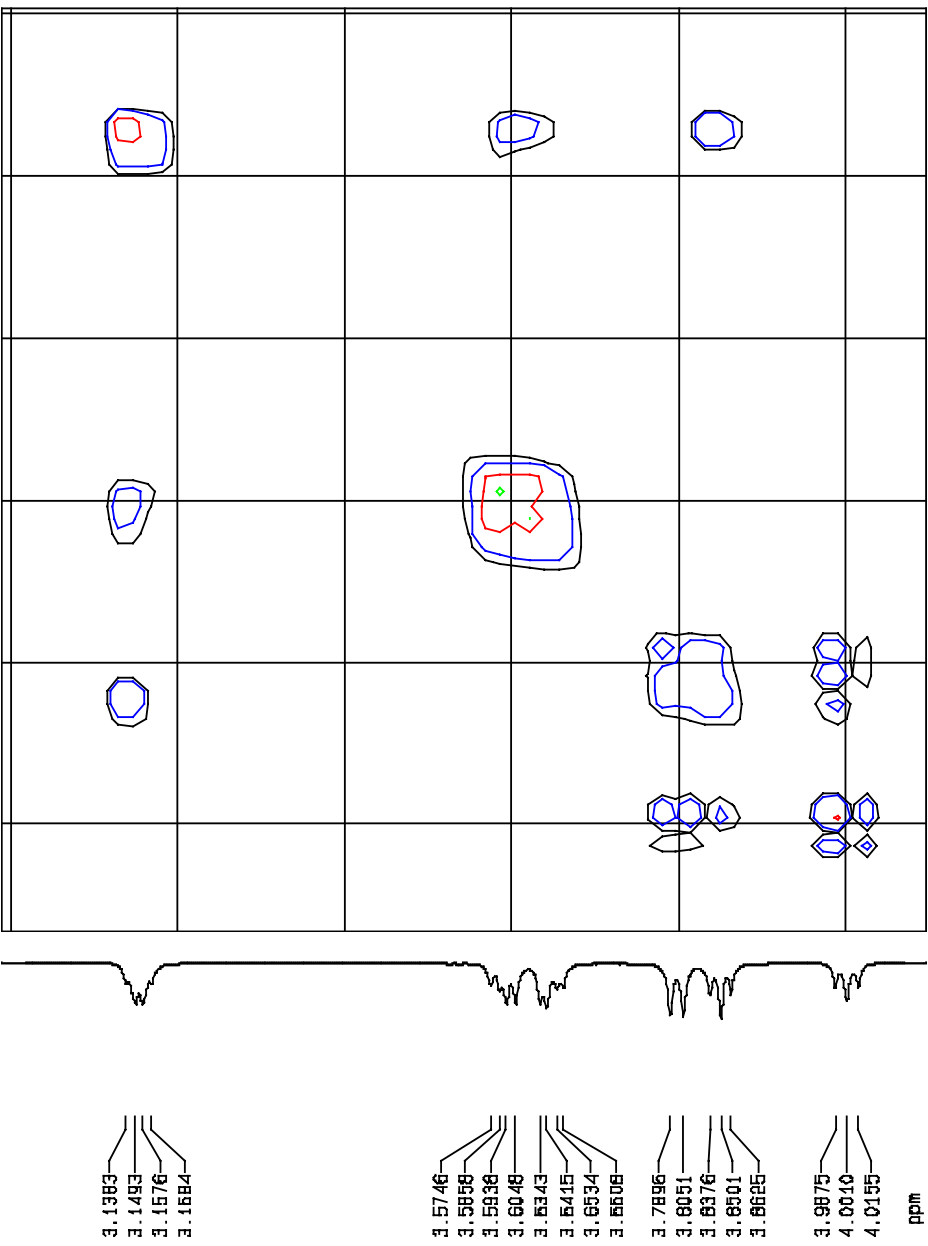

के 


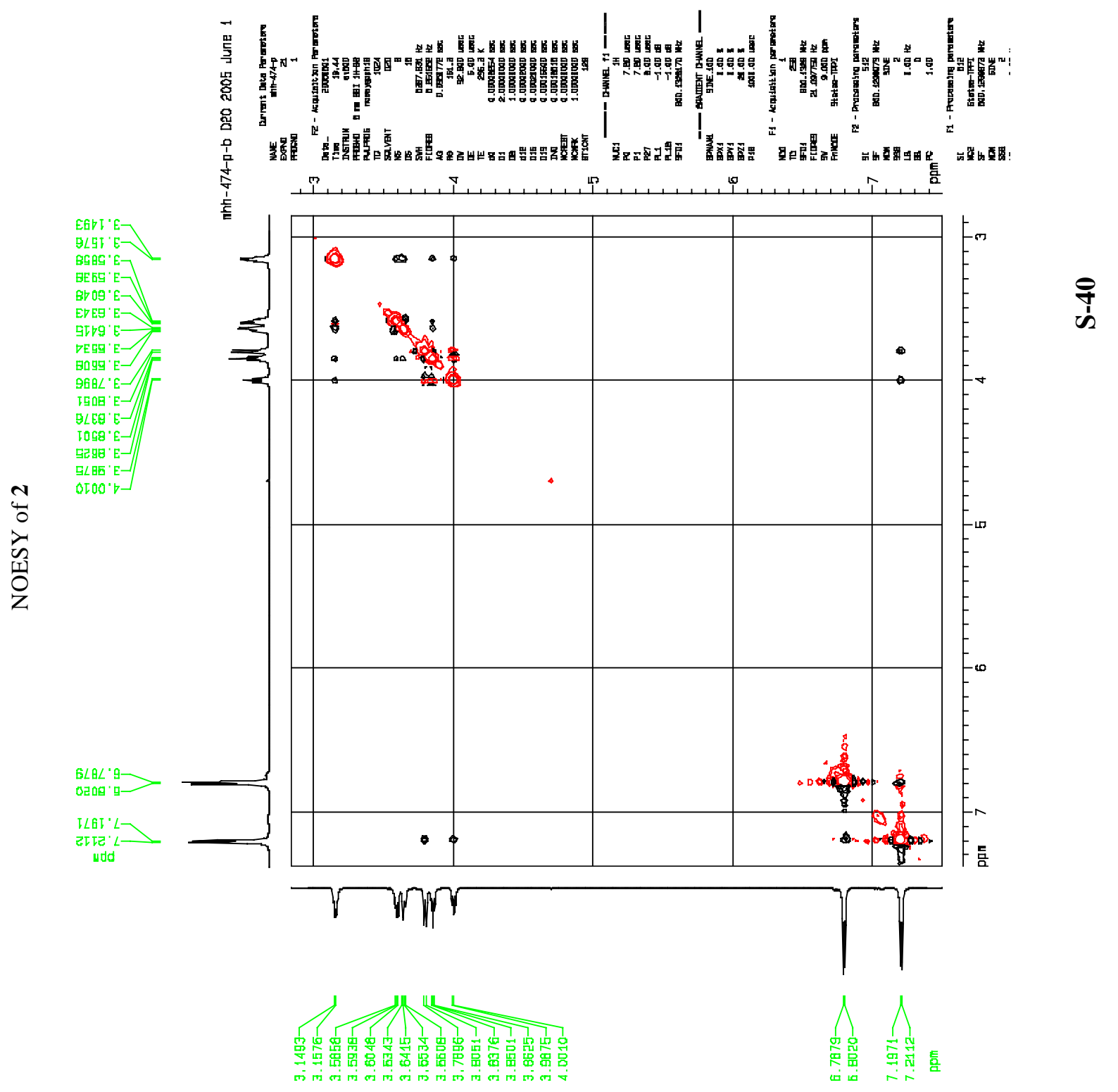




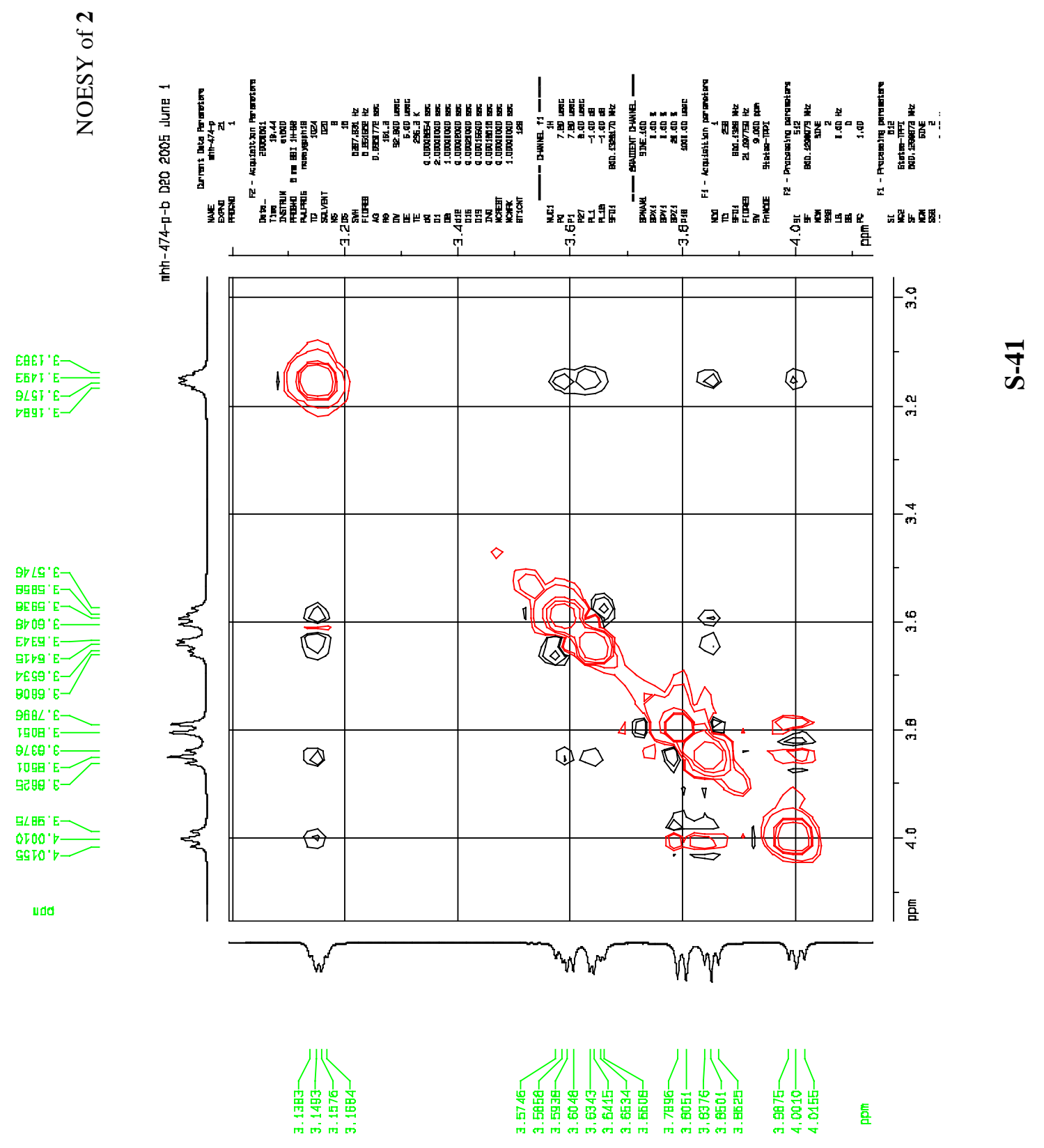




\section{Section D: $\quad$ References}

(1) Calimente, D. ; Postema , M.H.D. J.Org.Chem. 1999, 64, 1770-1771.

(2) (a) Cardona, F.; Faggi, E.; Liguori, F.; Cacciarini, M.; Goti, A. Tetrahedron Lett. 2003, 44, 2315-2318. (b) Desvergnes, S. ; Py, S ; Vallée, Y. J. Org. Chem. 2005, 70, 1459-1462.

(3) Yu Y.; Singh, S. K.; Liu, A.; Li,T.-K.; Liu L. F.; LaVoie E. J. Bioorg. Med. Chem. 2003, 11, 1475-1491.

(4) Fuji , K.; Ichikawa , K.; Node , M.; Fujita , E. J. Org. Chem. 1979 , 44, 1661-1664.

(5) Shibano, M. ; Tsukamoto, D. ; Masuda, A. ; Tanaka, Y. ; Kusano, G. Chem. Pharm. Bull., 2001, 49, 1362-1365. 